\title{
Integral Representation of Functions of Bounded Variation
}

\author{
Z. Lipcsey, ${ }^{1}$ I. M. Esuabana ${ }^{1},^{1}$ J. A. Ugboh, ${ }^{1}$ and I. O. Isaac ${ }^{2}$ \\ ${ }^{1}$ Department of Mathematics, University of Calabar, Nigeria \\ ${ }^{2}$ Department of Mathematics, Akwa Ibom State University, Nigeria
}

Correspondence should be addressed to I. M. Esuabana; esuabana@unical.edu.ng

Received 21 April 2019; Accepted 19 June 2019; Published 8 July 2019

Academic Editor: Ali Jaballah

Copyright (C) 2019 Z. Lipcsey et al. This is an open access article distributed under the Creative Commons Attribution License, which permits unrestricted use, distribution, and reproduction in any medium, provided the original work is properly cited.

\begin{abstract}
Functions of bounded variations form important transition between absolute continuous and singular functions. With Bainov's introduction of impulsive differential equations having solutions of bounded variation, this class of functions had eventually entered into the theory of differential equations. However, the determination of existence of solutions is still problematic because the solutions of differential equations is usually at least absolute continuous which is disrupted by the solutions of bounded variations. As it is known, if $f:\left[a, b_{\lambda}\right] \longrightarrow R^{n}$ is of bounded variation then $f$ is the sum of an absolute continuous function $f_{a}$ and a singular function $f_{s}$ where the total variation of $f_{s}$ generates a singular measure $\tau$ and $f_{s}$ is absolute continuous with respect to $\tau$. In this paper we prove that a function of bounded variation $f$ has two representations: one is $f$ which was described with an absolute continuous part with respect to the Lebesgue measure $\lambda$, while in the other an integral with respect to $\tau$ forms the absolute continuous part and $t(\tau)$ defines the singular measure. Both representations are obtained as parameter transformation images of an absolute continuous function on total variation domain $\left[a, b_{v}\right]$.
\end{abstract}

\section{Introduction}

Bainov, Laksmikantam, and Semionov introduced a new class of differential equations [1] which, in addition to the usual dynamics, had impulse effects, acting on the system in zero time periods. Hence, the dynamics was composed of the usual interactions and singular interactions. This led to solutions of bounded variations. As recent publications show, finding solutions of integral equations in the spaces of bounded variations in various fields is coming to the focus of attention of international research in mathematics like solution of Volterra-Hammerstein integral equations. This research was supported among others by the Venezuelan Central Bank [24].

The time dependence with the described composition of the dynamics led to difficulties in formulating efficient existence theorems, handling delay systems, and many other issues. The nature of bounded variation of the solutions was in contrast with such functions that constituted the solutions of differential equations (while not even being continuous).

We want to establish a presentation of the functions of bounded variation which makes it possible to handle the solutions of bounded variations in terms of absolute continuous functions. By the origin of the problem outlined, we will focus on $R^{n}$-valued functions of bounded variations. The combination of functions of bounded variation and singular functions in differential equations appears in the literature $[5,6]$.

It is well known that a function $f$ of bounded variation on an interval $\left[a, b_{\lambda}\right]$ is the sum of an absolute continuous component $f_{a}$ and a component $f_{s}$ singular with respect to the Lebesgue measure $\lambda[7,8]$. The total variation $\tau_{f_{s}}$ of $f_{s}$ is then an ascending singular function.

Let $\tau$ denote the measure defined by $\tau_{f_{s}}$ which is singular with respect to $\lambda$. Now we form a measure $v:=\lambda+\tau$ with respect to which both $\lambda \& \tau$ are absolute continuous. The measure $v$ is generated by the strictly ascending function $v(t):=t+\tau_{f}(t)$. The strictly ascending function $v$ has strictly ascending left and a right continuous versions $\nu_{\lambda,-}$ and $\nu_{\lambda,+}$, respectively [9], and maps the interval $\left[a, b_{\lambda}\right]$ into $\left[\nu(a), \nu\left(b_{\lambda}\right)\right]=:\left[a, b_{\nu}\right]$ where $b_{\nu}:=a+\bigvee_{a}^{b_{\lambda}} \nu$.

The transformation of $\left[a, b_{\gamma}\right]$ into $\left[a, b_{\lambda}\right]$ is defined as follows. The function $v$, being strictly ascending on $\left[a, b_{\lambda}\right]$, has a countable set $D_{\lambda} \subset\left[a, b_{\lambda}\right]$ of discontinuity points. Therefore, $\forall t \in D_{\lambda}$ the inequality $\nu(t-0)<v(t+0)$ holds, and $\nu$ is continuous in the rest of the points. Hence we define the 
transformation $\widehat{\mu}_{\lambda}$ as follows: $\widehat{\mu}_{\lambda}(t):=s$ if $\nu(s-0) \leq t \leq v(s+0)$ (see details in the paper).

These three transformations enable us to formulate and prove presentations stated in the main result. The theorem states that a function $f:\left[a, b_{\lambda}\right] \longrightarrow R^{n}$ is of bounded variation if and only if there exists an absolute continuous function $\varphi_{f}(u):=f(a)+\int_{a}^{u} \psi_{f} d v:\left[a, b_{v}\right] \longrightarrow R^{n}$ with $\psi_{f} \in L_{1}\left[\nu,\left[a, b_{\nu}\right], R^{n}\right]$ such that

$$
f(t)=\varphi_{f}\left(\mu_{\lambda,+}(t)\right)=f(a)+\int_{a}^{\mu_{\lambda,+}(t)} \psi_{f} d \nu .
$$

The same main theorem states that a function of bounded variation has another representation where measure $\tau$ gives the absolute continuous component and $t$ is an ascending function singular with respect to $\tau$.

\section{Integral Representation of Functions of Bounded Variation}

2.1. Summary of Known Results. See the details of this section in $[8,10]$.

Definition 1. A function $f:\left[a, b_{\lambda}\right] \longrightarrow R$ is a function of bounded variation if

$$
\bigvee_{a}^{b} f:=\sup _{\Phi \in \Gamma} \sum_{\substack{t_{j} \in \Phi \\ t j<b}}\left|f\left(t_{j+1}\right)-f\left(t_{j}\right)\right|<\infty,
$$

where $\Gamma:=\left\{\Phi \mid \Phi:=\left\{t_{j} \mid t_{j} \in\left[a, b_{\lambda}\right], a=t_{0}<t_{j}<t_{j+1}<\right.\right.$ $\left.\left.t_{k}=b, 0 \leq j<k\right\}, 1 \leq k<\infty\right\}$.

The main target of this paper is the study of ndimensional vector valued functions of bounded variation for some $n \geq 1$. We will introduce two types of variations for vector valued functions:

Definition 2. A function $f:\left[a, b_{\lambda}\right] \longrightarrow R^{n}$ is a function of bounded variation if

$$
\bigvee_{a}^{b} f:=\sup _{\Phi \in \Gamma} \sum_{\substack{t_{j} \in \Phi \\ t j<b}}\left\|f\left(t_{j+1}\right)-f\left(t_{j}\right)\right\|<\infty .
$$

Theorem 3. If $f:\left[a, b_{\lambda}\right] \longrightarrow R^{n}$ is a function ( $f=\left(f_{1}, f_{2}, \ldots\right.$ $\left.\left.f_{n}\right), f_{i}:\left[a, b_{\lambda}\right] \longrightarrow R, \forall 1 \leq i \leq n\right)$ then $\infty>\bigvee_{a}^{b} f \geq \bigvee_{a}^{b} f_{i}$, $\forall 1 \leq i \leq n$.

We will focus our investigations on the properties of ascending functions; therefore we will now turn to the integral properties of ascending functions of bounded variation (equivalent with boundedness on the interval under consideration).

2.2. Total Variation of a Function of Bounded Variation. We will first establish that ascending functions play major role in our integral representation of functions of bounded variations.
Theorem 4. Let $f:\left[a, b_{\lambda}\right] \longrightarrow R$ be a function of bounded variation. Then the function $\varphi_{f}(s):=\bigvee_{a}^{s} f, \forall s \in\left[a, b_{\lambda}\right]$ fulfils $\varphi_{f}(u)-\varphi_{f}(v) \geq f(u)-f(v), u \geq v, \forall u, v \in[a, b]$; hence $\varphi_{f}^{+}:=\varphi_{f}-f \geq 0$ is an ascending function and $f=\varphi_{f}-\varphi_{f}^{+}$.

Thus any function of bounded variation is differentiable almost everywhere and can be written as $f^{\prime}=d f / d \lambda$

$$
f_{a}(s):=f(a)+\int_{[a, s]} f^{\prime} d \lambda \quad \forall s \in[a, b]
$$

while $f_{s}:=f-f_{a}$ is the singular part of $f$. Hence $f=f_{a}+f_{s}$.

Proof. See $[8,10]$ for proof.

The singular function $f_{s}$ is of bounded variation; hence assume the following.

Definition 5. $\tau_{f}(s):=\bigvee_{a}^{s} f_{s}, \forall s \in\left[a, b_{\lambda}\right]$.

The singular function $f_{s}$ is absolute continuous with respect to $\tau_{f}$; hence $f_{s}(t)=\int_{a}^{t}\left(d f_{s} / d \tau_{f}\right) d \tau_{f}$ and therefore

$$
\begin{aligned}
f(t) & =f_{a}(a)+f_{s}(t) \\
& =f(a)+\int_{a}^{t} \frac{d f}{d \lambda} d \lambda+\int_{a}^{t} \frac{d f_{s}}{d \tau_{f}} d \tau_{f}, \quad \forall t \in\left[a, b_{\lambda}\right] .
\end{aligned}
$$

We introduce $\nu(t):=t+\tau_{f}(t), \forall t \in\left[a, b_{\lambda}\right]$ which is a strictly ascending function and $\nu:\left[a, b_{\lambda}\right] \longrightarrow\left[a, b_{\gamma}\right]$ with $b_{v}:=b+$ $\tau_{f}(b)$. It follows from Definition 5 of $\tau_{f}$ that

$$
\nu(s)=a+\bigvee_{a}^{s}\left(t+\tau_{f}\right), \quad \forall s \in\left[a, b_{\lambda}\right] .
$$

The function $v$ defines a measure $v:=\lambda+\tau$, such that both $\lambda \& \tau_{f}$ are absolute continuous with respect to $\nu$ :

$$
\begin{array}{ll}
\lambda(A)=\int_{A} \frac{d \lambda}{d \nu} d \nu, & \forall A \subset\left[a, b_{\lambda}\right] \text { Borel set, } \\
\tau(A)=\int_{A} \frac{d \tau}{d \nu} d \nu, \quad \forall A \subset\left[a, b_{\lambda}\right] \text { Borel set. }
\end{array}
$$

Using this in (5), we get an integral representation of the function $f$ of bounded variation in terms of a single measure:

$$
f(t)=f(a)+\int_{a}^{t}\left(\frac{d f}{d \lambda} \frac{d \lambda}{d \nu}+\frac{d f_{s}}{d \tau} \frac{d \tau}{d \nu}\right) d \nu
$$

2.2.1. Properties of the Radon-Nikodym Derivatives. The integral in (8) is a reformulation of (5) with the sum of two measures: $\lambda \& \tau$. This is a measure generated from a right continuous ascending function $\nu$. As stated in (7), both measures $\lambda \& \tau$ are absolute continuous with respect to $v$; therefore both can be written as an integral of the RadonNikodym derivatives [10] $\rho_{\lambda}=d \lambda / d \nu \& \rho_{\tau}=d \tau / d \nu$. These have the following important properties.

Lemma 6. Let $N_{\tau}:=\{d \tau / d \nu>0\} \& N_{\lambda}:=N_{\tau}^{\prime}$, and then

(1) $\lambda\left(N_{\tau}\right)=0=\tau\left(N_{\lambda}\right)$; 
(2) $\rho_{\lambda}+\rho_{\tau}=d(\lambda+\tau) / d \nu=d \tau / d \nu+d \lambda / d \nu=1$;

(3) $\rho_{\lambda}(x)=1, \forall x \in N_{\lambda}, \rho_{\tau}(x)=1, \forall x \in N_{\tau}$.

Proof. We call our attention to the following relation: let $A:=$ $\{(d \tau / d \nu)(d \lambda / d \nu)>0\}$. Then the singularity of $\tau$ with respect to $\lambda$ leads to the following relations:

$$
\begin{aligned}
\int_{A} \frac{d \tau}{d \nu} \frac{d \lambda}{d \nu} d \nu & =\int_{A} \frac{d \lambda}{d \nu} d \tau=\int_{\mathrm{A}} \frac{d \tau}{d \nu} d \lambda=\int_{A} \frac{d \tau}{d \lambda} \frac{d \lambda}{d \nu} d \lambda \\
& \leq \int_{A} \frac{d \tau}{d \lambda} d \lambda=0,
\end{aligned}
$$

since $\tau$ is singular with respect to $\lambda$ (if the fifth integral would be positive then A would be a set of positive $\nu$-measure with positive $\lambda \& \tau$ measures which contradicts the singularity of $\tau$ with respect to $\lambda)$. Hence $(d \tau / d \nu)(d \lambda / d \nu)=0$ almost everywhere with respect to $\nu$. Therefore $d \tau / d \lambda=0$ holds almost everywhere with respect to $\lambda$.

2.2.2. The Relationship between the $t$-Scale and the Total Variations Scale. This section presents the main construction of this paper which connects the structure of the domain of the function $f:\left[a, b_{\lambda}\right] \longrightarrow R$ of bounded variation and the range of the sum $v$ of the time parameter and the total variation $\tau$ of the singular component $f_{s}$ of $f$ defined in Definition 5.

The Structure from the Domain of a Function of Bounded Variation. The starting point here is the domain of the function $f:\left[a, b_{\lambda}\right] \longrightarrow R$ of bounded variation. The strictly ascending function $\nu(t)=t+\tau_{f}(t) \in\left[a, b_{\nu}\right]$ equivalently $\nu:=i d_{\left[a, b_{\lambda}\right]}+\tau_{f}:\left[a, b_{\lambda}\right] \longrightarrow\left[a, b_{\gamma}\right]$ with $b_{\nu}:=b_{\lambda}+\tau_{f}\left(b_{\lambda}\right)$ maps the domain $\left[a, b_{\lambda}\right]$ of $f$ to the range $\left[a, b_{\gamma}\right]$ of total variation scale. Both $i d_{\left[a, b_{\lambda}\right]} \& \tau_{f}$ are absolute continuous with respect to $v$ and the sets

$$
\begin{aligned}
N_{\lambda}: & =\left\{\frac{d i d_{[a, b]}}{d \nu}=1\right\} \\
\& N_{\tau}: & =\left\{\frac{d \tau}{d \nu}=1\right\}
\end{aligned}
$$

partition $\left[a, b_{\lambda}\right]$.

The function $v$ as a strictly ascending on $\left[a, b_{\lambda}\right]$ has its left and right continuous versions.

Definition 7.

$$
\begin{aligned}
\mu_{\lambda,-}(s) & :=v(s-0), \quad \forall s \in\left(a, b_{\lambda}\right) ; \\
\mu_{\lambda,-}(a) & :=a ; \\
\mu_{\lambda,-}\left(b_{\lambda}\right) & :=b_{\lambda} ; \\
\mu_{\lambda,+}(s) & :=v(s+0), \quad \forall s \in\left(a, b_{\lambda}\right) ; \\
\mu_{\lambda,+}(a) & :=a ; \\
\mu_{\lambda,+}\left(b_{\lambda}\right) & :=b_{\lambda} ;
\end{aligned}
$$

Definition 8. We assign a closed interval to each point of $\left[a, b_{\lambda}\right]: \emptyset \neq \Theta_{\lambda}(s):=\left[\mu_{\lambda,-}(s), \mu_{\lambda,+}(s)\right] \subset\left[a, b_{\gamma}\right]$ to each $s \in\left[a, b_{\lambda}\right]$.

Definition 9. Let $D_{\lambda}^{\nu}:=\bigcup_{t \in D_{f}} \Theta_{\lambda}(t)$ and let $\mathscr{D}_{\lambda}^{\nu}:=\left\{\Theta_{\lambda}(t) \mid\right.$ $\left.t \in D_{f}\right\} \subset \mathscr{P}\left(\left[a, b_{\nu}\right]\right)\left(\mathscr{P}\left(\left[a, b_{\nu}\right]\right)\right.$ denote the power set of $\left.\left[a, b_{\gamma}\right]\right)$.

Lemma 10. Both $\mu_{\lambda,-}, \mu_{\lambda_{,+}}:\left[a, b_{\lambda}\right] \longrightarrow\left[a, b_{\gamma}\right]$ are strictly ascending and $\mu_{\lambda,-}(a)=\mu_{\lambda,+}(a)=a \& \mu_{\lambda,-}\left(b_{\lambda}\right)=\mu_{\lambda,+}\left(b_{\lambda}\right)=$ $b_{v}$ by $a, b_{\lambda} \notin D_{f}$. Moreover both $\mu_{\lambda_{,-}}, \mu_{\lambda_{,+}}$are continuous on $\left[a, b_{\lambda}\right] \backslash D_{f} \longrightarrow\left[a, b_{\nu}\right] \backslash D_{\lambda}^{\nu}$.

The mapping defined in Definition 8 assigns a closed bounded interval to each point in $\left[a, b_{\lambda}\right]$ such that $\forall s, t \in$ $\left[a, b_{\lambda}\right], s \neq t \Longrightarrow \Theta_{\lambda}(s) \cap \Theta_{\lambda}(t)=\emptyset$ and $\bigcup_{s \in\left[a, b_{\lambda}\right]} \Theta_{\lambda}(s)=$ $\left[a, b_{\gamma}\right]$.

Proof.

(1) Both $\mu_{\lambda,-}, \mu_{\lambda,+}:\left[a, b_{\lambda}\right] \longrightarrow\left[a, b_{\gamma}\right]$ are strictly ascending and map $a \longrightarrow a \& b_{\lambda} \longrightarrow b_{\nu}$ by $a, b_{\lambda} \notin D_{f}$. Let $t, s \in\left[a, b_{\lambda}\right]$ be such that $t<s$. $\tau$ is an ascending function; hence $0 \leq \tau(s)-\tau(t) \Longrightarrow 0<(s+\tau(s))-$ $(t+\tau(t)) \Longrightarrow(t+\tau(t))<(s+\tau(s))$ that proves the first statement.

(2) By the proved strictly ascending property of $v$, $\forall u, s, v \in\left[a, b_{\lambda}\right], u<s<v \Longrightarrow \nu(u)<v(s)<v(v)$ holds. Hence $\sup \{v(u) \mid u<s\}=v(s-0) \leq v(s) \leq$ $v(s+0)=\inf \{v(u) \mid u>s\}$ implies that the proposed interval is the smallest interval enclosing the $v$-image of point $s$.

Moreover, if $t, s \in\left[a, b_{\lambda}\right], t \neq s \Longrightarrow$ either $t<w=$ $(t+s) / 2<s$ or $s<(t+s) / 2=w<t$ holds. Assume the first option. Then

$$
\begin{aligned}
& \Theta_{\lambda}(t) \subset[a, \inf \{v(u) \mid t<u\}] \\
& \subset {\left[a, v\left(\frac{w+t}{2}\right)\right) } \\
&\left(\nu\left(\frac{w+s}{2}\right), b\right] \supset[\sup \{v(u) \mid u<s\}, b] \supset \Theta_{\lambda}(s) \\
& \emptyset=\left[a, \frac{w+t}{2}\right) \cap\left(\frac{w+s}{2}, b\right] \\
& \supset \Theta_{\lambda}(t) \cap \Theta_{\lambda}(s) .
\end{aligned}
$$

(3) If $s \in\left[a, b_{\lambda}\right] \backslash D_{f}$ then $i d_{R}$ is continuous and $\tau$ is continuous since $s \notin D_{f}$; hence $v$ is continuous at $s$.

(4) Let $p \in\left[a, b_{\gamma}\right]$ then $p \in\left[a, b_{\gamma}\right] \backslash D_{\lambda}^{\nu}$ or $p \in D_{\lambda}^{\nu}$. In the first case $\exists s_{p} \in\left[a, b_{\lambda}\right] \backslash D_{\lambda}$ such that $p=\nu\left(s_{p}\right)$. In the second case by Definition $9 p \in D_{\lambda}^{\nu}:=\bigcup_{t \in D_{f}} \Theta_{\lambda}(t)$; hence $\exists$ ! $t_{p} \in D_{\lambda}$ such that $p \in \Theta_{\lambda}\left(t_{p}\right)$.

\section{Definition 11.}

$\widehat{\mu}_{\lambda}(u):=s$,

$\exists s \in\left[a, b_{\lambda}\right]$ such that $u \in \Theta_{\lambda}(s) \forall u \in\left[a, b_{\nu}\right]$. 
Lemma 12. The mapping $\widehat{\mu}_{\lambda}$ is constant on $\Theta_{\lambda}(s), \forall s \in[a, b]$, i. e., $\widehat{\mu}_{\lambda}(u)=\widehat{\mu}_{\lambda}(v), \forall u, v \in \Theta_{\lambda}(s), \forall s \in\left[a, b_{\lambda}\right]$. Moreover $\widehat{\mu}_{\lambda}$ : $\left[a, b_{\gamma}\right] \longrightarrow\left[a, b_{\lambda}\right]$ is continuous, ascending, and onto.

Proof. By (13) for $u, v \in \Theta_{\lambda}(s)$, it follows that $v(s-0) \leq u, v \leq$ $v(s+0) \Longrightarrow \widehat{\mu}_{\lambda}(u)=\widehat{\mu}_{\lambda}(v)=s$. The onto property follows from the proof of point (4) in Lemma 10.

Lemma 13. The relationships between $\mu_{\lambda_{,-},}, \mu_{\lambda_{,}+} \& \widehat{\mu}_{\lambda}$ are as follows:

$$
\begin{aligned}
& \widehat{\mu}_{\lambda} \circ \mu_{\lambda,-}=i d_{\left[a, b_{\lambda}\right]} ; \\
& \widehat{\mu}_{\lambda} \circ \mu_{\lambda,+}=i d_{\left[a, b_{\lambda}\right]} .
\end{aligned}
$$

However, exchanging the two mappings will not give $i d_{\left[a, b_{v}\right]}$. Indeed,

$$
\begin{aligned}
& \mu_{\lambda,-} \circ \widehat{\mu}_{\lambda}=i d_{\left[a, b_{\nu}\right] \backslash D_{\lambda}^{v}} ; \\
& \mu_{\lambda,+} \circ \widehat{\mu}_{\lambda}=i d_{\left[a, b_{v}\right] \backslash D_{\lambda}^{\nu}} .
\end{aligned}
$$

Proof. We prove it for the first case of (14) only. Let $s \in\left[a, b_{\lambda}\right]$. Then $\mu_{\lambda,-}(s)=\nu(s-0)=\nu(s)$ if $s \notin D_{f} \cdot \widehat{\mu}_{\lambda}(\nu(s))=s$ since $\Theta_{\lambda}(s)=\{\nu(s)\}$ (when $s$ is a continuity point, $\Theta_{\lambda}(s)$ is a one point set by Definition 11).

If $s \in D_{f}$ then $\mu_{\lambda,-}(s)=\nu(s-0)$ and $\nu(s-0) \in \Theta_{\lambda}(s)$ the left endpoint of which is $\mu_{\lambda,-}$ (s) (by the definition of $\Theta_{\lambda}$ in Lemma 10). Similarly, if $s \in D_{f}$ then $\mu_{\lambda,+}(s)=v(s+0)$ and $\nu(s+0) \in \Theta_{\lambda}(s)$ the right endpoint of which is $\mu_{\lambda,+}(s)$ (by the definition of $\Theta_{\lambda}$ in Lemma 10). Hence the first statement in (14) of the lemma is proved.

We now prove the first statement of (15). Let $s \in\left[a, b_{\gamma}\right] \backslash$ $D_{\lambda}^{\nu}$. Then $\widehat{\mu}_{\lambda}(s) \in\left[a, b_{\lambda}\right] \backslash D_{f}$ since $s \notin D_{\lambda}^{\nu}$; hence $s \in \Theta_{\lambda}(u)$ for a continuity point $u \in\left[a, b_{\lambda}\right] \backslash D_{f}$ of $\nu$. And $\widehat{\mu}_{\lambda}(s)=u \Longrightarrow$ $\mu_{\lambda,-}(u)=s$. This proves the lemma.

The partitioning of $\left[a, b_{\lambda}\right]$ into the union $N_{\lambda} \cup N_{\tau}$ can be transformed into the partitioning of $\left[a, b_{\nu}\right]$ as follows.

\section{Definition 14.}

$$
\begin{aligned}
& N_{\lambda}^{v}=\widehat{\mu}_{\lambda}^{-1}\left(N_{\lambda}\right) \\
& N_{\tau}^{v}=\widehat{\mu}_{\lambda}^{-1}\left(N_{\tau}\right)
\end{aligned}
$$

The Structure from the Total Variation. In this section we will construct functions of bounded variations from the scale $\left[a, b_{\gamma}\right]$ and show that the time interval $\left[a, b_{\lambda}\right], \tau, N_{\lambda}, N_{\tau}$, is obtainable from the structure defined below.

Definition 15. Let $\left[a, b_{\gamma}\right]$ be a closed bounded interval and let $\left\{\left[a, b_{\nu}\right], \mathscr{B}_{\nu,\left[a, b_{\nu}\right]}, \nu\right\}$ be the Lebesgue measure defined by $i d_{\left[a, b_{v}\right]}:\left[a, b_{\nu}\right] \stackrel{\longrightarrow}{\longrightarrow}\left[a, b_{\nu}\right]$ on the Borel sets $\mathscr{B}_{\nu,\left[a, b_{\nu}\right]}$ of $\left[a, b_{\nu}\right]$. Let $N_{\lambda}^{v}, N_{\tau}^{v} \subset\left[a, b_{\nu}\right]$ be Borel subsets of $\left[a, b_{v}\right]$ such that

$$
\nu\left(N_{\lambda}^{\nu}\right) \times \nu\left(N_{\tau}^{\nu}\right)>0
$$

(where non of them is of zero measure)

$$
\begin{aligned}
& N_{\lambda}^{v} \cap N_{\tau}^{v}=\emptyset ; \\
& N_{\lambda}^{v} \cup N_{\tau}^{v}=\left[a, b_{\nu}\right] .
\end{aligned}
$$

Since the conditions prescribed for time and $\tau$ in Definition 15 are symmetric, we will construct two representations with mappings below.

Definition 16. The definition of basic mappings for time representation is as follows:

(1) let the time scale interval be $\left[a, b_{\lambda}\right]$ with $b_{\lambda}:=a+$ $\int_{a}^{b_{v}} \chi_{N_{\lambda}^{v}} d v$

(2) $\widehat{\mu}_{\lambda}(s):=a+\int_{a}^{s} \chi_{N_{\lambda}^{\nu}} d \nu \in\left[a, b_{\lambda}\right], \forall s \in\left[a, b_{\nu}\right]$;

(3) $\mu_{\lambda,-}(s):=\inf \widehat{\mu}_{\lambda}^{-1}(\{s\}) \in\left[a, b_{\gamma}\right], \forall s \in\left[a, b_{\lambda}\right]$;

(4) $\mu_{\lambda}(s):=\mu_{\lambda,+}(s):=\sup \widehat{\mu}_{\lambda}^{-1}(\{s\}) \in\left[a, b_{v}\right], \forall s \in\left[a, b_{\lambda}\right]$.

Definition 17. The definition of the $\tau$-based mappings for $\tau$ representation is as follows:

(1) let the $\tau$-scale interval be $\left[a, b_{\tau}\right]$ with $b_{\tau}:=a+$ $\int_{a}^{b_{v}} \chi_{N_{\tau}^{v}} d v$

(2) $\widehat{\mu}_{\tau}(s):=a+\int_{a}^{s} \chi_{N_{\tau}^{v}} d \nu \in\left[a, b_{\tau}\right], \forall s \in\left[a, b_{\nu}\right]$;

(3) $\mu_{\tau,-}(s):=\inf \widehat{\mu}_{\tau}^{-1}(\{s\}) \in\left[a, b_{\gamma}\right] ; \forall s \in\left[a, b_{\tau}\right]$;

(4) $\mu_{\tau}(s):=\mu_{\tau,+}(s):=\sup \widehat{\mu}_{\tau}^{-1}(\{s\}) \in\left[a, b_{\gamma}\right], \forall s \in\left[a, b_{\tau}\right]$.

We will treat the time case only since the other is the same word for word.

(1) The Time, Singular Time $\tau$ and $v$

(1) If $\widehat{\mu}_{\lambda}(s)=u, \widehat{\mu}_{\lambda}(t)=v \in\left[a, b_{\lambda}\right] \& u<v \Longrightarrow s<$ $t \forall s, t \in\left[a, b_{\gamma}\right]$ by Definition 16 (2) (if the integral on an interval is positive then the length of the interval is positive.).

(2) By the axiom of choice, $\exists v:\left[a, b_{\lambda}\right] \longrightarrow\left[a, b_{\gamma}\right]$ is a strictly ascending function such that $v(t) \in$ $\widehat{\mu}_{\lambda}^{-1}(t), \forall t \in\left[a, b_{\lambda}\right]$. Then $\mu_{\lambda,-}(s)=v(s-$ 0) \& $\mu_{\lambda,+}(s)=\nu(s+0), \forall s \in\left[a, b_{\lambda}\right]$ which follows from the continuity of $\widehat{\mu}_{\lambda}$.

(3) The ascending function $v:\left[a, b_{\lambda}\right] \longrightarrow\left[a, b_{\nu}\right]$ has a countable set of discontinuity points $D_{\lambda}:=\{t \mid v(t-$ $0)<v(t+0)\}$. Hence $v:\left[a, b_{\lambda}\right] \backslash D_{\lambda} \longrightarrow\left[a, b_{v}\right]$ is continuous.

(4) By ascending continuity of $\widehat{\mu}_{\lambda}$ (absolute continuous for being an indefinite integral) $\Theta_{\lambda}(t):=\widehat{\mu}_{\lambda}^{-1}(\{t\})$ is closed bounded interval by being preimage of a closed singleton $\forall t \in\left[a, b_{\lambda}\right]$. Moreover $\Theta_{\lambda}(t)=$ $\left[\mu_{\lambda,-}(t), \mu_{\lambda,+}(t)\right] \forall t \in\left[a, b_{\lambda}\right]$ and $\Theta_{\lambda}(t) \cap \Theta_{\lambda}(s)=$ $\emptyset, \forall t, s \in\left[a, b_{\lambda}\right], t \neq s$.

(5) The integral representation of $\nu(t), t \in\left[a, b_{\lambda}\right]$ : using Definition $16(2)$ of $\widehat{\mu}_{\lambda}(s):=a+\int_{a}^{s} \chi_{N_{\lambda}^{\nu}} d \nu \in\left[a, b_{\lambda}\right]$, 
$\forall s \in\left[a, b_{\nu}\right]$ and the definition of $\nu$ in point (2) gives the right continuous version of $\nu$, i.e.,

$$
\begin{aligned}
v(t) & =a+\int_{a}^{\nu(t+0)} 1 d \nu=a+\int_{a}^{\nu(t+0)}\left(\chi_{N_{\lambda}^{\nu}}+\chi_{N_{\tau}^{v}}\right) d \nu \\
& =\int_{a}^{\nu(t+0)} \chi_{N_{\lambda}^{v}} d \nu+\int_{a}^{\nu(t+0)} \chi_{N_{\tau}^{v}} d \nu \\
t & :=\int_{a}^{\nu(t+0)} \chi_{N_{\lambda}^{\nu}} d \nu \\
\tau(t) & :=\int_{a}^{\nu(t+0)} \chi_{N_{\tau}^{v}} d \nu \\
\nu(t) & =t+\tau(t) \quad \forall t \in\left[a, b_{\lambda}\right] .
\end{aligned}
$$

Here we used the relations in (17) and defined $\tau$ : $\left[a, b_{\lambda}\right] \longrightarrow\left[0, b_{\nu}-b_{\lambda}\right]$ as ascending mapping.

(6) The ascending function $\tau$ is singular with respect to $\lambda$ and vice versa, and the ascending function $\lambda$ is singular with repect to $\tau$. Let $u, v \in\left[a, b_{\lambda}\right] \backslash D_{\lambda}$. Then

$$
\int_{\nu(u)}^{\nu(v)} \frac{d \tau}{d \nu} \frac{d \lambda}{d \nu} d \nu=\int_{\nu(u)}^{\nu(v)} \chi_{N_{\tau}^{v}} \chi_{N_{\lambda}^{\nu}} d \nu=0
$$

(7) Hence for any $\varphi, \psi \in L_{1}\left(\left[a, b_{\lambda}\right], v\right)$

$$
\begin{aligned}
f(t) & :=f(a)+\int_{a}^{t} \varphi \chi_{N_{\lambda}} d \nu+\int_{a}^{t} \psi \chi_{N_{\tau}} d \nu \\
& =f(a)+\int_{a}^{t} \varphi d \lambda+\int_{a}^{t} \psi d \tau
\end{aligned}
$$

is a function of bounded variation.

With this we proved that the two representations mutually determine each other and what is more there are two representations of any function of bounded variation which is not absolute continuous.

2.2.3. $v$-Integrable Functions on Time and $v$-Scales. In this section we will construct everything for the $t / \lambda$ scale. All steps applied here can be done for the $\tau$ scale also.

Since the ascending functions $\tau \& \nu$ are noncontinuous functions of $t$, the $\tau / \nu$ measures have to be defined from the semiring of left closed, right open intervals with continuity endpoints.

Definition 18. Let the semiring of left closed, right open intervals with endpoints of continuity in $\left[a, b_{\lambda}\right]$ be

$$
\mathscr{P}_{\nu,\left[a, b_{\lambda}\right], c}:=\left\{[u, v) \mid u, v \in\left[a, b_{\lambda}\right] \backslash D_{\lambda}\right\}
$$

Let the smallest $\sigma$-algebra containing the semiring be

$$
\mathscr{B}_{\nu,\left[a, b_{\lambda}\right], c}:=\sigma\left(\mathscr{P}_{\nu,\left[a, b_{\lambda}\right], c}\right) .
$$

Definition 19. Let the semiring of left closed right open intervals with endpoints of continuity in $\left[a, b_{\tau}\right]$ be

$$
\mathscr{P}_{\nu,\left[a, b_{\tau}\right], c}:=\left\{[u, v) \mid u, v \in\left[a, b_{\tau}\right] \backslash D_{\tau}\right\}
$$

Let the smallest $\sigma$-algebra containing the semiring be

$$
\mathscr{B}_{\nu,\left[a, b_{\tau}\right], c}:=\sigma\left(\mathscr{P}_{\nu,\left[a, b_{\tau}\right], c}\right)
$$

Definition 20. Let the semiring of left closed right open intervals with endpoints from $\left[a, b_{\gamma}\right] \backslash D_{\lambda}^{\nu}$ in $\left[a, b_{\gamma}\right]$ for $\lambda$ be

$$
\mathscr{P}_{\lambda,\left[a, b_{\nu}\right], c}^{\nu}:=\left\{[u, v) \mid u, v \in\left[a, b_{\nu}\right] \backslash D_{\lambda}^{\nu}\right\}
$$

and the semiring of left closed right open intervals with endpoints from $\left[a, b_{\gamma}\right] \backslash D_{\tau}^{v}$ in $\left[a, b_{\nu}\right]$ for $\tau$ be

$$
\mathscr{P}_{\tau,\left[a, b_{\nu}\right], c}^{\nu}:=\left\{[u, v) \mid u, v \in\left[a, b_{\nu}\right] \backslash D_{\tau}^{\nu}\right\}
$$

The corresponding $\sigma$-algebras are defined as follows.

Definition 21. Let the smallest $\sigma$-algebra containing the semiring $\mathscr{P}_{\lambda,\left[a, b_{v}\right], c}^{\nu}$ be

$$
\mathscr{B}_{\lambda,\left[a, b_{\nu}\right], c}^{\nu}:=\sigma\left(\mathscr{P}_{\lambda,\left[a, b_{\nu}\right], c}^{\nu}\right)
$$

and the smallest $\sigma$-algebra containing the semiring $\mathscr{P}_{\tau,\left[a, b_{y}\right], c}^{v}$ be

$$
\mathscr{B}_{\tau,\left[a, b_{\nu}\right], c}^{\nu}:=\sigma\left(\mathscr{P}_{\tau,\left[a, b_{\nu}\right], c}^{\nu}\right)
$$

Now we can define the measures from the ascending function $v$ and from the ascending function $i d_{\left[a, b_{y}\right]}$.

Definition 22.

$$
\begin{array}{ll}
\nu_{\lambda}([s, t)):=v(t)-v(s) & \forall[s, t) \in \mathscr{P}_{\nu,\left[a, b_{\lambda}\right], c} \\
\nu_{\tau}([s, t)):=v(t)-v(s) & \forall[s, t) \in \mathscr{P}_{\nu,\left[a, b_{\tau}\right], c}
\end{array}
$$

Definition 23.

$$
\begin{array}{ll}
\nu_{\lambda}^{\nu}([s, t)):=t-s \quad \forall[s, t) \in \mathscr{P}_{\lambda,\left[a, b_{\nu}\right], c}^{\nu} \\
\nu_{\tau}^{\nu}([s, t)):=t-s \quad \forall[s, t) \in \mathscr{P}_{\tau,\left[a, b_{\nu}\right], c}^{\nu}
\end{array}
$$

An important relationship is presented in the following lemma.

Lemma 24. From $\mathscr{P}_{\lambda,\left[a, b_{\nu}\right], c}^{\nu} \subset \mathscr{P}\left(\left[a, b_{\nu}\right], \nu\right)$ it follows that $\mathscr{B}_{\lambda,\left[a, b_{\nu}\right], c}^{\nu} \subset \mathscr{B}\left(\left[a, b_{\nu}\right], \nu\right)$.

From $\mathscr{P}_{\tau,\left[a, b_{\nu}\right], c}^{\nu} \subset \mathscr{P}\left(\left[a, b_{\nu}\right], v\right)$ it follows that $\mathscr{B}_{\tau,\left[a, b_{\nu}\right], c}^{\nu} \subset$ $\mathscr{B}\left(\left[a, b_{v}\right], v\right)$.

Moreover $\nu_{\lambda}^{\nu}(A)=\nu(A), \forall A \in \mathscr{B}_{\lambda,\left[a, b_{\nu}\right], c}^{\nu}$ and $\nu_{\tau}^{\nu}(A)=$ $\nu(A), \forall A \in \mathscr{B}_{\tau,\left[a, b_{v}\right], c}^{\nu}$. 
Proof. Let $A \in \mathscr{B}_{\lambda,\left[a, b_{\nu}\right], c}^{\nu}$. By the statement of this theorem $\mathscr{P}_{\lambda,\left[a, b_{\nu}\right], c}^{\nu} \subset \mathscr{P}\left(\left[a, b_{\nu}\right], v\right) \subset \mathscr{B}\left(\left[a, b_{\nu}\right], \nu\right)$. Then

$$
\begin{aligned}
& v_{\lambda}^{\nu}(A)=\nu_{\lambda}^{\nu *}(A)=\inf \left\{\sum_{j=0}^{\infty} v\left(\left[a_{j}, b_{j}\right)\right) \mid A\right. \\
& \subset \bigcup_{j=0}^{\infty}\left[a_{j}, b_{j}\right),\left\{\left[a_{j}, b_{j}\right)\right\}_{j=1}^{\infty} \subset \mathscr{P}_{\lambda,\left[a, b_{\nu}\right], c}^{\nu} \\
& \left.\subset \mathscr{B}\left(\left[a, b_{\nu}\right], \nu\right)\right\} .
\end{aligned}
$$

Let us select a sequence $\left\{S_{k}\right\}_{k=1}^{\infty}$ from the covers of $A$ constructed in (32) as follows:

$$
\begin{gathered}
\left\{S_{k} \mid S_{k}:=\left\{\left[a_{k, j}, b_{k, j}\right)\right\}_{j=1}^{\infty}, A \subset \bigcup_{j=0}^{\infty}\left[a_{k, j}, b_{k, j}\right), \frac{1}{2^{k}}\right. \\
\left.\left.>\sum_{j=0}^{\infty} v\left(\left[a_{k, j}, b_{k, j}\right)\right)-v_{\lambda}^{\nu *}(A)\right)\right\} .
\end{gathered}
$$

Lemma 25. Let $\left\{I_{t} \mid I_{t} \in \mathscr{P}_{\lambda,\left[a, b_{y}\right], c}^{\nu}, \quad \forall 1 \leq t<\infty\right\}$ and let $H:=\bigcup_{t=1}^{\infty} I_{t}$. Then $\exists\left\{D_{s}\right\}_{s=1}^{\infty} \subset \mathscr{R}\left(\mathscr{P}_{\lambda,\left[a, b_{v}\right], c}^{v}\right)$ such that $H=\bigcup_{s=1}^{\infty} D_{s}, \forall 1 \leq n, m<\infty, n \neq m, D_{n} \cap D_{m}=\emptyset$.

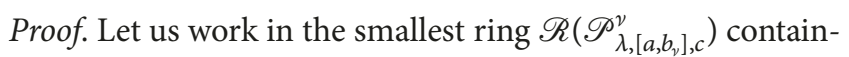
ing the semiring $\mathscr{P}_{\lambda,\left[a, b_{\nu}\right], c}^{\nu}$. Since $I_{t} \in \mathscr{P}_{\lambda,\left[a, b_{\nu}\right], c}^{\nu} \Longrightarrow I_{t} \in$ $\mathscr{R}\left(\mathscr{P}_{\lambda,\left[a, b_{y}\right], c}^{v}\right) \forall 1 \leq t<\infty$. Let $D_{u}:=I_{u} \backslash\left(\bigcup_{q=1}^{u-1} I_{q}\right) \in$ $\mathscr{R}\left(\mathscr{P}_{\lambda,\left[a, b_{y}\right], c}^{v}\right), \forall 1 \leq u<\infty$ such that $H=\bigcup_{q=1}^{\infty} D_{q}$ and $D_{n} \cap D_{m}=\emptyset, \forall 1 \leq n, m<\infty, n \neq m$, where we used the standard technique of changing an infinite union of ring elements into infinite union of pairwise disjoint ring elements $[8,11]$.

Corollary 26. By the representation theorem of $\mathscr{R}\left(\mathscr{P}_{\lambda,\left[a, b_{\nu}\right], c}^{\nu}\right)$, $\exists$ a pairwise disjoint system $\left\{D_{j, i}\right\}_{i=1}^{k_{j}} \subset \mathscr{P}_{\lambda,\left[a, b_{v}\right], c}^{v}$ such that $D_{j}=\bigcup_{i=1}^{k_{j}} D_{j, i}$. Hence $H=\left(\bigcup_{q=1}^{\infty} D_{q}\right)=$ $\left(\bigcup_{q=1}^{\infty}\left(\bigcup_{i=1}^{k_{q}} D_{q, i}\right)\right)$. Therefore $H$ is obtained as a countable pairwise disjoint union of left closed right open intervals with continuity endpoints. Hence $H \in \mathscr{B}\left(\left[a, b_{\gamma}\right], v\right)$ and $\nu_{\lambda}^{\nu}(H)=$ $\nu(H)$. (33).

Let us apply these propositions to the sequence defined in

Let $A_{k}:=\bigcup_{j=1}^{\infty}\left[a_{k, j}, b_{k, j}\right) \supset A$ and let $B_{k}:=\bigcap_{j=1}^{k} A_{j} \supset A$, $\forall 1 \leq k<\infty$. By the conditions of the theorem, $\mathscr{P}_{\lambda,\left[a, b_{y}\right], c}^{\nu} \subset$ $\mathscr{P}\left(\left[a, b_{v}\right], v\right) \subset \mathscr{B}\left(\left[a, b_{\nu}\right], v\right) \Longrightarrow \forall 0<k \in \mathbb{N}, A_{k}, B_{k} \in$ $\mathscr{B}_{\lambda,\left[a, b_{v}\right], c}^{\nu} \& A_{k}, B_{k} \in \mathscr{B}\left(\left[a, b_{\nu}\right], \nu\right)$.
Then let $B:=\bigcap_{k=1}^{\infty} B_{k} \in \mathscr{B}_{\lambda,\left[a, b_{\nu}\right], c}^{v}$ and

$$
\begin{aligned}
\nu_{\lambda}^{\nu}(B \backslash A) & =\nu_{\lambda}^{\nu}(B)-v_{\lambda}^{\nu}(A)<v_{\lambda}^{\nu}\left(B_{n}\right)-\nu_{\lambda}^{\nu}(A) \\
& \left.<\sum_{j=1}^{\infty} v\left(\left[a_{k, j}, b_{k, j}\right)\right)-v_{\lambda}^{\nu *}(A)\right)<\frac{1}{2^{k}},
\end{aligned}
$$

$\forall k \in \mathbb{N}$

where we used the definition of the covering intervals in (33). Hence $B \backslash A \in \mathscr{B}_{\lambda,\left[a, b_{\nu}\right], c}^{\nu}, \& \nu_{\lambda}^{\nu}(B \backslash A)=0 \Longrightarrow 0=\nu_{\lambda}^{\nu}(B \backslash A)=$ $\nu_{\lambda}^{\nu *}(B \backslash A) \geq \nu^{*}(B \backslash A)=0$. Recalling that any set of 0 outer measure is measurable we get $B \backslash A \in \mathscr{B}\left(\left[a, b_{\nu}\right], v\right)$, hence with $B \in \mathscr{B}\left(\left[a, b_{\nu}\right], v\right) \Longrightarrow A \in \mathscr{B}\left(\left[a, b_{\gamma}\right], v\right)$.

(1) The Relationship between $\lambda$ or $\tau$ Structures and $\nu$-Ones. We will show that $\mathscr{P}_{\nu,\left[a, b_{\lambda}\right], c} \& \mathscr{P}_{\lambda,\left[a, b_{\nu}\right], c}^{\nu}$ and $\mathscr{P}_{\nu,\left[a, b_{\tau}\right], c} \& \mathscr{P}_{\tau,\left[a, b_{\nu}\right], c}^{\nu}$ are isomorphic semirings, respectively, and what is more, the isomorphisms preserve the respective measures.

\section{Lemma 27.}

$$
\begin{aligned}
\mu_{\lambda,-}([u, v)) & =[v(u-0), v(v-0)) \\
& =[v(u), v(v)) \in \mathscr{P}_{\lambda,\left[a, b_{v}\right], c}^{v} ; \\
v_{\lambda}^{v}\left(\mu_{\lambda,-}([u, v))\right) & =v_{\lambda}^{v}([v(u), v(v)))=v(v)-v(u) \\
& =v_{\lambda}([u, v)) .
\end{aligned}
$$

by $u, v \in\left[a, b_{\lambda}\right] \backslash D_{\lambda} \Longrightarrow \nu(u), v(v) \in\left[a, b_{\nu}\right] \backslash D_{\lambda}^{v}$.

Conversely, let $[s, t) \in \mathscr{P}_{\lambda,\left[a, b_{\nu}\right], c}$ and then

$$
\begin{aligned}
\widehat{\mu}_{\lambda}([s, t)) & =\left[\widehat{\mu}_{\lambda}(s), \widehat{\mu}_{\lambda}(t)\right) \in \mathscr{P}_{\nu,\left[a, b_{\lambda}\right], c} ; \\
v_{\lambda}\left(\widehat{\mu}_{\lambda}([s, t))\right) & \left.=v_{\lambda}\left(\left[\widehat{\mu}_{\lambda}(s)\right), \widehat{\mu}_{\lambda}(t)\right)\right) \\
& =v\left(\widehat{\mu}_{\lambda}(s)\right)-v\left(\widehat{\mu}_{\lambda}(t)\right)=t-s \\
& =v_{\lambda}^{v}([s, t))
\end{aligned}
$$

by the identity $\nu\left(\widehat{\mu}_{\lambda}\right)=i d_{\left[a, b_{\nu}\right] \backslash D_{\lambda}^{v}}$ in (15) and Definition 23.

Proof. Essentially the lemma contains the references from which the statements of the lemma follow.

2.2.4. The Relationships between the Semirings and Their Extensions. The lemma below describes the relationship between the continuity points of $\nu$ and the points in $\left[a, b_{\lambda}\right]$.

Lemma 28. $\forall t \in\left[a, b_{\lambda}\right] \exists\left\{l_{t, j}\right\}_{j=1}^{\infty},\left\{u_{t, j}\right\}_{j=1}^{\infty} \subset\left[a, b_{\lambda}\right] \backslash D_{\lambda}$ such that

(1) if $a=t \Longrightarrow l_{t, j}=a, \forall 1 \leq j<\infty$; otherwise if $a<t$ then $l_{t, j}<l_{t, j+1}, \forall 0<j \in \mathbb{N}$ and if $t=b \Longrightarrow u_{t, j}=b, \forall 1 \leq j<\infty$; otherwise if $t<b$ then $u_{t, j}>u_{t, j+1}, \forall 0<j \in \mathbb{N}$;

(2) $l_{t, j} \nearrow$ t for $j \longrightarrow \infty$ and $u_{t, j} \searrow$ for $j \longrightarrow \infty$;

(3) $v\left(l_{t, j}\right) \nearrow \mu_{\lambda,-}(t)=v(t-0)$ for $j \longrightarrow \infty$ and $v\left(u_{t, j}\right) \searrow$ $\mu_{\lambda,+}(t)=\nu(t+0)$ for $j \longrightarrow \infty$. 
Proof.

(1) Let $t=a$, then $l_{a, j}:=a, \forall 1 \leq j<\infty$. Similarly, if $t=b$ then $u_{b, j}:=b, \forall 1 \leq j<\infty$.

(2) Let $t \in(a, b)$. Let $U:=a<t<b=: V \& j=1$.

(3) Since $D_{\lambda}$ is countable and the open intervals $\emptyset \neq$ $(U, t) \&(t, V) \nsubseteq D_{\lambda} \Longrightarrow \exists p \in(U, t) \backslash D_{\lambda} \& q \epsilon$ $(t, V) \backslash D_{\lambda}$ (continuity points).

(4) Let $l_{t, j}:=p \& u_{t, j}:=q$.

(5) Let $U:=\max \{(p+t) / 2,(U+t) / 2\}, \& V:=\min \{(q+$ $t) / 2,(V+t) / 2\}$ and $j=j+1$.

(6) Go to (3).

(7) This process will stop after generating the sequences of continuity points which are strictly ascending/descending sequences converging to $t$.

By the strictly ascending property of $\nu$ on $\left[a, b_{\lambda}\right] \backslash D_{\lambda}$ the sequences $\left\{v\left(l_{t, j}\right)\right\}_{j=1}^{\infty} \&\left\{\nu\left(u_{t, j}\right)\right\}_{j=1}^{\infty}$ converge to left and right limits of $\nu$ at $t \in\left[a, b_{\lambda}\right]$, respectively. This completes the proof.

\section{Lemma 29.}

(1) $\forall s, t \in\left[a, b_{\lambda}\right], s<t$ the following statements hold:

(a) $[s, t] \in \mathscr{B}_{\lambda,\left[a, b_{\lambda}\right], c}$;

(b) $\left[\mu_{\lambda,-}(s), \mu_{\lambda,+}(t)\right]=[\nu(s-0), \nu(t+0)] \in \mathscr{B}_{\lambda,\left[a, b_{\nu}\right], c^{\prime}}^{\nu}$

(2) $\forall A \in \mathscr{D}_{\lambda}^{\nu} \Longrightarrow A \in \mathscr{B}_{\lambda,\left[a, b_{v}\right], c}^{\nu}$ and $A$ is an atom which means $\forall B \in \mathscr{B}_{\lambda,\left[a, b_{y}\right], c}^{v}$ either $B \subset A \Longrightarrow B=\emptyset$ or $B \supseteq$ A. Equivalently a measurable set does not cut an atom:

$$
A \cap B=\left\{\begin{array}{ll}
A & \text { or } \\
\emptyset &
\end{array} .\right.
$$

(3) If $f$ is $\mathscr{B}_{\lambda,\left[a, b_{1}\right], c}^{v}$-measurable then $\forall A \in \mathscr{D}_{\lambda}^{\nu}, f(s)=$ $c_{A}, \forall s \in A$, where $c_{A} \in R$ is a constant depending on $A$.

(4) An interval $[s, t] \in \mathscr{B}_{\lambda,\left[a, b_{v}\right], c}^{\nu}$ is in $\mathscr{D}_{\lambda}^{\nu}$ if and only if $\widehat{\mu}_{\lambda}([s, t])=[u, v] \subset N_{\tau} \& \rho_{\tau}(s)=1 \& \rho_{\lambda}(s)=0 \forall s \in$ $[u, v]$.

Proof.

(1) By Lemma $28 \forall s, t \in\left[a, b_{\lambda}\right], s<t, \exists\left\{l_{s, j}\right\}_{j=1}^{\infty} \subset\left[a, b_{\lambda}\right] \backslash$ $D_{\lambda}, \& \exists\left\{u_{t, j}\right\}_{j=1}^{\infty} \subset\left[a, b_{\lambda}\right] \backslash D_{\lambda}$ such that the following applies.

(a) If $a<s$ then $\left\{l_{s, j}\right\}_{1 \leq j<\infty}$ is strictly ascending and $l_{s, j} \nearrow s$ for $j \longrightarrow \infty$. For $a=s$ the sequence is constant $a$.

(b) If $t<b$ then $\left\{u_{t, j}\right\}_{1 \leq j<\infty}$ is strictly descending and $u_{t, j} \searrow t$ for $j \longrightarrow \infty$. For $b=t$ the sequence is constant $b$. (c) By $\left[l_{s, j}, u_{t, j}\right) \in \mathscr{P}_{\nu,\left[a, b_{\lambda}\right], c} \subset \mathscr{B}_{\lambda,\left[a, b_{\lambda}\right], c} \forall 1 \leq$ $j<\infty$ it follows that $[s, t]=\bigcap_{j=1}^{\infty}\left[l_{s, j}, u_{t, j}\right) \in$ $\mathscr{B}_{\lambda,\left[a, b_{\lambda}\right], c}$ (similarly for the intervals with closed $b$ ending).

(d) $\lim _{j \rightarrow \infty} v\left(l_{s, j}\right)=v(s-0)$ and $\lim _{j \rightarrow \infty} v\left(u_{t, j}\right)=$ $v(t+0)$.

(e) Hence $\left[v\left(l_{s, j}\right), v\left(u_{t, j}\right)\right) \in \mathscr{P}_{\lambda,\left[a, b_{\gamma}\right], c}^{v} \subset \mathscr{B}_{\lambda,\left[a, b_{\nu}\right], c}^{v}$ and

$$
\bigcap_{j=1}^{\infty}\left[v\left(l_{s, j}\right), v\left(u_{t, j}\right)\right)=[v(s-0), v(t+0)]
$$

by (d) of this proof. Hence from $\mathscr{B}_{\lambda,\left[a, b_{y}\right], c}^{\nu}$ being $\sigma$-algebra it follows that

$$
[\nu(s-0), \nu(t+0)] \in \mathscr{B}_{\lambda,\left[a, b_{\gamma}\right], c}^{\nu} .
$$

(2) Let $A \in \mathscr{D}_{\lambda}^{\nu} \Longrightarrow A=\Theta_{\lambda}(t)$ for a $t \in D_{\lambda}$. Hence $\Theta_{\lambda}(t)=[\nu(t-0), \nu(t+0)] \in \mathscr{B}_{\lambda,\left[a, b_{\nu}\right], c}^{v}$ by point (1) of this proof. We show now that if $H \subset A$ then $H$ is not measurable.

Let $p \in A \in \mathscr{D}_{\lambda}^{\nu} \Longrightarrow A=\Theta_{\lambda}(t)=[\nu(t-0), \nu(t+0)]$ for a $t \in D_{\lambda}$. If $s \in\left[a, b_{v}\right] \backslash D_{\lambda}^{v} \subset\left[a, b_{v}\right] \backslash A \Longrightarrow p \in[u, v) \in$ $\mathscr{P}_{v,\left[a, b_{\lambda}\right], c} \Longrightarrow u, v \notin A \Longrightarrow u<v(t-0)<v(t+0)<v$ by $u, v \in\left[a, b_{v}\right] \backslash A$. Hence $\emptyset \neq H \subset A \Longrightarrow \exists p \in$ $H \Longrightarrow p \in A$. Then $[u, v) \in \mathscr{P}_{v,\left[a, b_{\lambda}\right], c} \Longrightarrow v_{\lambda}^{v}(A) \leq$ $\nu_{\lambda}^{\nu *}(H)$ holds for the outer measure of $H$. Hence $\mathrm{H}$ is not measurable if $H \neq A$ by Caratheodory's condition for outer measures $\left(\nu_{\lambda}^{\nu * *}(Y)=\nu_{\lambda}^{\nu * *}(Y \cap H)+\nu_{\lambda}^{\nu *}(Y \backslash\right.$ $\left.H), \forall Y \subset\left[a, b_{v}\right]\right)$ with $Y=A$ :

$\nu_{\lambda}^{\nu *}(A)<2 \times \nu_{\lambda}^{\nu *}(A)<\nu_{\lambda}^{\nu *}(A \cap H)+\nu_{\lambda}^{\nu *}(A \backslash H)$

proves the lemma.

(3) Let $f:\left[a, b_{v}\right] \longrightarrow R$ be measurable with respect to $\mathscr{B}_{\lambda,[a, b], c}^{\nu}$. Let us assume that $\exists A \in \mathscr{D}_{\lambda}^{\nu}$ and $\exists s, t \in A$ such that $f(s) \neq f(t)$. Then with $\alpha:=(f(s)+f(t)) / 2$ the sets $U:=\{f>\alpha\} \cap A \neq \emptyset \& V:=\{f<\alpha\} \cap A \neq \emptyset$ are disjoint and hence proper nonempty subsets of the measurable set $A$; hence by statement 1 of this lemma, the sets $U \& V$ are not measurable; hence $\mathrm{f}$ is not measurable. If $f:\left[a, b_{\nu}\right] \longrightarrow R^{n}$ is measurable and then each component of $\mathrm{f}$ is measurable and hence constant on the elements of $\mathscr{D}_{\lambda}^{\nu}$; hence the statement follows.

(4)

(a) Let $[s, t] \in \mathscr{B}_{\lambda,\left[a, b_{\nu}\right], c}^{v} \cap \mathscr{D}_{\lambda}^{\nu}$. Then $\exists u \in D_{\lambda}$ such that $[s, t]=\Theta_{\lambda}(u)$. Then by Definition 16 point (2) $u=\widehat{\mu}_{\lambda}(v):=u+\int_{s}^{v} \chi_{N_{\lambda}^{v}} d v, \forall v \in[s, t]$. Hence $0=\int_{s}^{t} \chi_{N_{\lambda}^{v}} d v, \Longrightarrow \chi_{N_{\lambda}^{v}}=0$ almost everywhere. By statements (2) and (3) of this lemma, the measurable function $\chi_{N_{\lambda}^{v}}=$ const on $[s, t]$ follows $\chi_{N_{\lambda}^{v}}=0$ in $[s, t]$; hence $\chi_{N_{\tau}^{v}}=1$ in $[s, t]$. 
(b) Conversely, let $[s, t] \in \mathscr{B}_{\lambda,\left[a, b_{v}\right], c}^{v}$ and let $\chi_{N_{\tau}^{v}}(v)=$ $1, \forall v \in[s, t]$. Then $\chi_{N_{1}^{v}}(v) \stackrel{=}{=}, \forall v \in[s, t]$. Hence by Definition 16 point (2) $u=\widehat{\mu}_{\lambda}(v):=$ $u+\int_{s}^{v} \chi_{N_{\lambda}^{v}} d v=u+\int_{s}^{v} 0 d v, \forall v \in[s, t]$. Hence $\Theta_{\lambda}(u)=[s, t]$ proves that $[s, t] \in \mathscr{D}_{\lambda}^{\nu}$.

\section{Lemma 30.}

Case (1). Let $s, t \in\left[a, b_{\lambda}\right], s<t$, and let $\chi_{[s, t]}:\left[a, b_{\lambda}\right] \longrightarrow\{0,1\}$ fulfil $\chi_{[s, t]}(u)=1$ if $u \in[s, t]$ and 0 otherwise. Then $\chi_{[s, t]} \circ \widehat{\mu}_{\lambda}=$ $\chi_{\left[\nu_{\lambda,-}(s), \nu_{\lambda,+}(t)\right]}:\left[a, b_{\nu}\right] \longrightarrow\{0,1\}$.

Case (2). Let $s, t \in\left[a, b_{\gamma}\right] \backslash D_{\lambda}^{\nu}, s<t$ and let $\chi_{[s, t]}:\left[a, b_{\gamma}\right] \longrightarrow$ $\{0,1\}$ fulfil $\chi_{[s, t]}(u)=1$ if $u \in[s, t]$ and 0 otherwise. Then $\chi_{[s, t]} \circ \mu_{\lambda,-}=\chi_{\left[\hat{v}_{\lambda}(s), \hat{\nu}_{\lambda}(t)\right]}=\chi_{\left[v_{\lambda,-}^{-1}(s), \nu_{\lambda,+}^{-1}(s)\right]}:\left[a, b_{\lambda}\right] \longrightarrow\{0,1\}$.

Proof.

Case (1). $\left\{u \mid \chi_{[s, t]} \circ \widehat{\mu}_{\lambda}(u)=1\right\}=\left\{u \mid s \leq \widehat{\mu}_{\lambda}(u) \leq t\right\}$. Applying Lemma 13 and the set of equations (14) to the left and right sides of the inequalities, respectively, leads to the following:

$$
\begin{aligned}
s & \leq \widehat{\mu}_{\lambda}(u) \mid \circ v_{\lambda,-} \Longleftrightarrow v_{\lambda,-}(s) \leq u \\
\widehat{\mu}_{\lambda}(u) & \leq t \mid \circ v_{\lambda,+} \Longleftrightarrow u \leq v_{\lambda,+}(t) .
\end{aligned}
$$

Hence $\left\{u \mid s \leq \widehat{\mu}_{\lambda}(u) \leq t\right\}=\left\{u \mid v_{\lambda,-}(s) \leq u \leq v_{\lambda_{,-}}(t)\right\}$ has been proved and therefore case (1) holds.

Case (2). Let $s, t \in\left[a, b_{v}\right] \backslash D_{\lambda}^{\nu}, s<t$. Then $\left\{u \mid \chi_{[s, t]} \circ \mu_{\lambda,-}(u)=\right.$ $1\}=\left\{u \mid s \leq \mu_{\lambda,-}(u) \leq \mu_{\lambda,+}(u) \leq t\right\}$. Applying Lemma 13 and the set of equations (15) leads to the following:

$$
\begin{aligned}
s & \leq \mu_{\lambda,-}(u) \mid \circ \widehat{\mu}_{\lambda} \Longleftrightarrow \widehat{\mu}_{\lambda}(s) \leq u \\
\mu_{\lambda,+}(u) & \leq t \mid \circ \widehat{\mu}_{\lambda} \Longleftrightarrow u \leq \widehat{\mu}_{\lambda}(t) .
\end{aligned}
$$

Hence $\left\{u \mid s \leq \mu_{\lambda,-}(u) \leq \mu_{\lambda,+}(u) \leq t\right\}=\left\{u \mid \widehat{\mu}_{\lambda}(s) \leq u \leq\right.$ $\left.\widehat{\mu}_{\lambda}(t)\right\}$ has been proved and therefore case (2) holds.

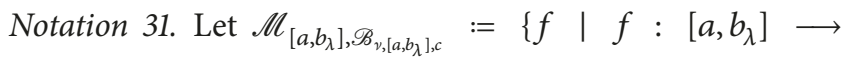

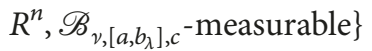

$$
\begin{aligned}
& \mathscr{M}_{\left[a, b_{\nu}\right], \mathscr{B}_{\nu,\left[a, b_{\lambda}\right], c}^{v}}^{\nu} \\
& \quad=\left\{f \mid f:\left[a, b_{\nu}\right] \longrightarrow R^{n}, \mathscr{B}_{\lambda,\left[a, b_{\nu}\right], c}^{\nu} \text {-measurable }\right\}
\end{aligned}
$$

2.2.5. Transpose of the Transformations between $t$ and Total Variation Scales. Let us consider the transpose of a transformation of the domains of function spaces.

Definition 32. Let $A, B$ \& $C$ be sets and let $A \stackrel{\varphi}{\rightarrow} B \stackrel{h}{\rightarrow} C$ with $h \in\{\psi: B \longrightarrow C\}=: \mathscr{F}(B, C)$ and let $\mathscr{F}(A, C):=\{\psi: A \longrightarrow$ $C\}$. Then let

$$
\varphi^{*}: h \in \mathscr{F}(B, C) \longrightarrow h \circ \varphi \in \mathscr{F}(A, C),
$$

$\forall h \in \mathscr{F}(B, C)$.

Note that $\mathscr{F}(B, C) \stackrel{\varphi^{*}}{\longrightarrow} \mathscr{F}(A, C)$ against $A \stackrel{\varphi}{\rightarrow} B$.
Notation 33. Notations for the lemma about the transpose mappings. Let $A:=\left[a, b_{\lambda}\right] \& B:=\left[a, b_{\gamma}\right]$ and let $C:=R^{n}$. Let $\mathscr{F}[A: C]:=\left\{f:\left[a, b_{\lambda}\right] \longrightarrow R^{n}\right\}=: \mathscr{F}\left[\left[a, b_{\lambda}\right]: R^{n}\right]$ be a set of functions.

Let $\mathscr{F}[B \quad: \quad C]:=\left\{\begin{array}{lllll}B & f & : & {\left[a, b_{\nu}\right]} & \longrightarrow\end{array}\right.$ $R^{n}$ such that $f$ is constant on any $\left.A \in \mathscr{D}_{\lambda}^{\nu}\right\}=: \mathscr{F}\left[\left[a, b_{\nu}\right]\right.$ : $\left.R^{n}\right]$,

Lemma 34. Let $\widehat{\mu}_{\lambda}:\left[a, b_{\gamma}\right] \longrightarrow\left[a, b_{\lambda}\right]$ and $\mu_{\lambda,-}:\left[a, b_{\lambda}\right] \longrightarrow$ $\left[a, b_{\nu}\right]$ be two mappings as defined above. Then the following relations hold:

$$
\begin{aligned}
& \widehat{\mu}_{\lambda}^{*} \circ \mu_{\lambda,-}^{*}=i d_{\mathscr{F}\left[\left[a, b_{y}\right]: R^{n}\right]} \\
& \mu_{\lambda,-}^{*} \circ \widehat{\mu}_{\lambda}^{*}=i d_{\mathscr{F}\left[\left[a, b_{\lambda}\right]: R^{n}\right]}
\end{aligned}
$$

Moreover $\widehat{\mu}_{\lambda}^{*} \& \mu_{\lambda,-}^{*}$ are linear isomorphisms.

Proof.

(1) Let $f \in \mathscr{F}\left[\left[a, b_{y}\right]: R^{n}\right]$ and let $\mu_{\lambda_{2}-}^{*}$ be the transpose of $\mu_{\lambda,-}:\left[a, b_{\lambda}\right] \longrightarrow\left[a, b_{\nu}\right]$. Then

$$
\mu_{\lambda,-}^{*} f(s)=f\left(\mu_{\lambda,-}(s)\right), \quad \forall s \in\left[a, b_{\lambda}\right] .
$$

(2) Let $g \in \mathscr{F}\left[\left[a, b_{\lambda}\right]: R^{n}\right]$ and let $\widehat{\mu}_{\lambda}^{*}$ be the transpose of $\widehat{\mu}_{\lambda}:\left[a, b_{v}\right] \longrightarrow\left[a, b_{\lambda}\right]$. Then

$$
\widehat{\mu}_{\lambda}^{*} g(v)=g\left(\widehat{\mu}_{\lambda}(v)\right), \quad \forall v \in\left[a, b_{\nu}\right] .
$$

(3) Let us consider now the composition $\widehat{\mu}_{\lambda}^{*} \circ \mu_{\lambda,-}^{*} f$ :

$\widehat{\mu}_{\lambda}^{*} \circ \mu_{\lambda,-}^{*} f=\widehat{\mu}_{\lambda}^{*}\left(f \circ \mu_{\lambda,-}\right)=f \circ\left(\mu_{\lambda,-} \circ \widehat{\mu}_{\lambda}\right)$.

By the first identity in (15) $\mu_{\lambda,-} \circ \widehat{\mu}_{\lambda}=i d_{\left[a, b_{v}\right] \backslash D_{\lambda}^{\nu}}$ we discovered that $\forall u \in\left[a, b_{v}\right] \backslash D_{\lambda}^{v} \Longrightarrow \widehat{\mu}_{\lambda}^{*} \circ \mu_{\lambda,-}^{*} f(u)=$ $f(u)$ holds.

If $u \in D_{\lambda}^{v}$ then $u \in \Theta_{\lambda}(t)$ for a $t \in D_{\lambda}$; hence $f\left(\mu_{\lambda,-}(t)\right)=f(u), u, \mu_{\lambda,-}(t) \in \Theta_{\lambda}(t)$; hence $f(u)=$ $f\left(\mu_{\lambda,-}(t)\right)=$ const $_{(t, f)}$ (with $\mathrm{f}$ being constant on $\left.\Theta_{\lambda}(t)\right)$.

By (13) $\widehat{\mu}_{\lambda}\left(\Theta_{\lambda}(t)\right)=\{t\} \Longrightarrow \forall u \in \Theta_{\lambda}(t) \Longrightarrow f(u)=$ $f\left(\mu_{\lambda,-}\left(\widehat{\mu}_{\lambda}(u)\right)\right)=f\left(\mu_{\lambda,-}(t)\right)=$ const $_{(t, f)}$ completes the proof.

(4) Let us now consider the composition $\mu_{\lambda,-}^{*} \circ \widehat{\mu}_{\lambda}^{*} g$ :

$$
\begin{aligned}
\mu_{\lambda,-}^{*} \circ \widehat{\mu}_{\lambda}^{*} g & =\mu_{\lambda,-}^{*}\left(g \circ \widehat{\mu}_{\lambda}\right)=g \circ\left(\mu_{\lambda,-} \circ \widehat{\mu}_{\lambda}\right) \\
& =g \circ i d_{\left[a, b_{\lambda}\right]}
\end{aligned}
$$

by (14). Hence $\widehat{\mu}_{\lambda}^{*} g\left(\mu_{\lambda,-}(u)\right)=g\left(\mu_{\lambda,-}\left(\widehat{\mu}_{\lambda}(s)\right)\right)=$ $g(u) \forall u \in\left[a, b_{\lambda}\right]$. 
(5) If $R^{n}$ is a vector space then $\mathscr{F}\left[\left[a, b_{\lambda}\right]: R^{n}\right] \&$ $\mathscr{F}\left[\left[a, b_{\tau}\right]: R^{n}\right]$ are vector spaces for the point-wise addition and multiplication by scalar. Equation (45) gives the linear isomorphism.

Corollary 35. Let the notations of Lemma 34 be changed as follows:

$$
\begin{aligned}
& \mathscr{F}\left[\left[a, b_{\lambda}\right]: R^{n}\right] \longrightarrow \mathscr{M}_{\left[a, b_{\lambda}\right], \mathscr{B}_{v,\left[a, b_{\lambda}\right], c}} \\
& \mathscr{F}\left[\left[a, b_{\gamma}\right]: R^{n}\right] \longrightarrow \mathscr{M}_{\left[a, b_{\nu}\right], \mathscr{R}_{\nu,\left[a, b_{\lambda}\right], c}^{v}}^{v}
\end{aligned}
$$

Then Lemma 34 with

$$
\begin{aligned}
& \widehat{\mu}_{\lambda}^{*}: \mathscr{M}_{\left[a, b_{\lambda}\right], \mathscr{B}_{v,\left[a, b_{\lambda}\right], c}} \longrightarrow \mathscr{M}_{\left[a, b_{\nu}\right], \mathscr{B}_{v,\left[a, b_{\lambda}\right], c}^{v}}^{v} \text { and } \\
& \text { Precisely } \\
& \widehat{\mu}_{\lambda}^{*} \circ \mu_{\lambda,-}^{*}=i d_{\mathscr{M}_{\left[a, b_{\lambda}\right], \mathscr{P}_{\nu,\left[a, b_{\lambda}\right], c}}} \\
& \mu_{\lambda,-}^{*} \circ \widehat{\mu}_{\lambda}^{*}=i d_{\mathscr{M}_{\left[a, b_{\nu}\right], \mathscr{V}_{v,\left[a, b_{\lambda}\right], c}^{v}}}
\end{aligned}
$$$$
\mu_{\lambda,-}^{*}: \mathscr{M}_{\left[a, b_{v}\right], \mathscr{B}_{v,\left[a, b_{1}\right], c}^{v}}^{*} \longrightarrow \mathscr{M}_{\left[a, b_{\lambda}\right], \mathscr{B}_{v,\left[a, b_{\gamma}\right], c}} \text { holds. }
$$

Hence the mappings $\mu_{\lambda,-} \& \widehat{\mu}_{\lambda}$ are linear isomorphisms between the two measurable function spaces.

Proof. The two continuous ascending mappings $\mu_{\lambda_{1}-}$ : $\left[a, b_{\lambda}\right] \longrightarrow\left[a, b_{\gamma}\right] \& \widehat{\mu}_{\lambda}:\left[a, b_{\nu}\right] \longrightarrow\left[a, b_{\lambda}\right]$ between the two domains $\left[a, b_{\lambda}\right] \&\left[a, b_{\gamma}\right]$ are the same as in Lemma 34 .

Let $f \in \mathscr{M}_{\left[a, b_{y}\right], \mathscr{B}_{v,\left[a, b_{\lambda}\right], c}^{v}}^{v} f$ be measurable and $\mu_{\lambda,-}$ is continuous; hence

$$
\mu_{\lambda,-}^{*} f=f \circ \mu_{\lambda,-} \in \mathscr{M}_{\left[a, b_{\lambda}\right], \mathscr{B}_{\nu,\left[a, b_{\lambda}\right], c}^{v}} .
$$

Let $g \in \mathscr{M}_{\left[a, b_{\lambda}\right], \mathscr{B}_{2,\left[a, b_{\lambda}\right], c}}$ be measurable and $\widehat{\mu}_{\lambda}$ is continuous; hence

$\widehat{\mu}_{\lambda}^{*} g=g \circ \widehat{\mu}_{\lambda} \in \mathscr{M}_{\left[a, b_{\nu}\right], \mathscr{B}_{\nu,\left[a, b_{\lambda}\right], c}}$. By the properties proved in Lemma $34 \widehat{\mu}_{\lambda}^{*} g$ is constant on all $A \in \mathscr{D}_{\lambda}^{\nu}$. Hence the statements of Lemma 34 are true (the two identities in (45)).

Let $L_{1}\left[v_{\lambda},\left[a, b_{\lambda}\right], c\right]:=\left\{f \mid f:\left[a, b_{\lambda}\right] \longrightarrow R\right.$ be $\nu_{\lambda}$-integrable $\}$ and let

$L_{1}\left[v_{\lambda}^{v},\left[a, b_{\nu}\right], c\right]:=\left\{f \mid f:\left[a, b_{v}\right] \longrightarrow R\right.$ be $\nu_{\lambda}^{v}$-integrable\}. Then the following applies.

Theorem 36. By Corollary 35 it follows that

$$
\begin{aligned}
& \widehat{\mu}_{\lambda}^{*} \circ \mu_{\lambda,-}^{*}=i d_{L_{1}\left[v_{\lambda}^{*},\left[a, b_{\nu}\right], c\right]} \\
& \mu_{\lambda,-}^{*} \circ \widehat{\mu}_{\lambda}^{*}=i d_{L_{1}\left[\nu_{\lambda},\left[a, b_{\lambda}\right], c\right]}
\end{aligned}
$$

What is more,

$$
\begin{aligned}
\int_{a}^{b_{\lambda}} f d \nu_{\lambda}=\int_{a}^{b_{\nu}} \widehat{\mu}_{\lambda}^{*} f d \nu_{\lambda}^{\nu}, & \\
& \forall f \in L_{1}\left[\nu_{\lambda},\left[a, b_{\lambda}\right], c\right] ; \\
\int_{a}^{b_{\lambda}} \mu_{\lambda,-}^{*} f d \nu_{\lambda}=\int_{a}^{b_{\nu}} f d \nu_{\lambda}^{v} & \forall f \in L_{1}\left[\nu_{\lambda}^{\nu},\left[a, b_{\nu}\right], c\right]
\end{aligned}
$$

Proof.

(1) By Definition 22, let $[s, t) \in \mathscr{P}_{\nu,\left[a, b_{\lambda}\right], c}$ be a measurable set and let its characteristic function be $\chi_{[s, t)}$. Then

$$
\begin{aligned}
\nu_{\lambda}([s, t)) & :=v(t)-\nu(s)=\int_{a}^{b_{\lambda}} \chi_{[s, t)} d \nu_{\lambda} \\
& =\int_{a}^{b_{\nu}} \widehat{\mu}_{\lambda}^{*} \chi_{[s, t)} d \nu_{\lambda}^{\nu}
\end{aligned}
$$

By Definition 23 let $[s, t) \in \mathscr{P}_{\lambda,[a, b, y, c}^{\nu}$ be a measurable set and let its characteristic function be $\chi_{[s, t)}$. Then

$$
\nu_{\lambda}^{\nu}([s, t)):=t-s=\int_{a}^{b_{\lambda}} \mu_{\lambda,-}^{*} \chi_{[s, t)} d \nu_{\lambda}=\int_{a}^{b_{\nu}} \chi_{[s, t)} d \nu_{\lambda}^{\nu}
$$

(2) By Lemma 34 for any subset $A \subset\left[a, b_{\lambda}\right] \Longrightarrow \widehat{\mu}_{\lambda}^{*} A \subset$ $\left[a, b_{\gamma}\right]$ and

$$
\left(\nu_{\lambda}\right)^{*}(A)=\left(\nu_{\lambda}^{\nu}\right)^{*}\left(\widehat{\mu}_{\lambda}^{*} A\right)
$$

holds for the outer measures $\left(\nu_{\lambda}\right)^{*} \&\left(\nu_{\lambda}^{\nu}\right)^{*}$.

Conversely $\forall A \subset\left[a, b_{\nu}\right]$ such that $\forall H \in \mathscr{D}_{\lambda}^{\nu}$ either $H \cap A=\emptyset$ or $H \subset A$ and then the relation holds: $\left(\nu_{\lambda}\right)^{*}\left(\mu_{\lambda,-}^{*} A\right)=\left(\nu_{\lambda}^{\nu}\right)^{*}(A)$.

From this it follows that for measurable sets in both $\sigma$-algebras the outer measures define the extended measures and therefore the measures on the $\sigma$ algebras are fulfilled. This is

$$
\begin{array}{ll}
v_{\lambda}^{\nu}(A)=\int_{a}^{b_{\lambda}} \mu_{\lambda,-}^{*} \chi_{A} d \nu_{\lambda}=\int_{a}^{b_{\nu}} \chi_{A} d \nu_{\lambda}^{\nu}, & \\
\forall A \in \mathscr{B}_{\lambda,\left[a, b_{\nu}\right], c}^{\nu} \\
\nu_{\lambda}(A)=\int_{a}^{b_{\lambda}} \chi_{A} d \nu_{\lambda}=\int_{a}^{b_{\nu}} \widehat{\mu}_{\lambda}^{*} \chi_{A} d \nu_{\lambda}^{\nu},
\end{array}
$$

$$
\forall A \in \mathscr{B}_{\nu,\left[a, b_{\lambda}\right], c}
$$

By the linearity of the transpose mappings, the identities are valid for all nonnegative step-functions and from there for all integrable function. This completes the proof of the theorem.

Theorem 37. Let $f \in L_{1}\left[v_{\lambda},\left[a, b_{\lambda}\right], c\right]$ \& $t \in\left[a, b_{\lambda}\right]$. Then

$$
\int_{a}^{t} f d \nu_{\lambda}=\int_{a}^{\mu_{\lambda,+}(t)} \widehat{\mu}_{\lambda}^{*} f d \nu_{\lambda}^{\nu}
$$

Let $f \in L_{1}\left[\nu_{\lambda}^{\nu},\left[a, b_{\nu}\right], c\right] \&[a, s] \in \mathscr{B}_{\lambda,\left[a, b_{\nu}\right], c}^{\nu}$ and $s \in\left[a, b_{\nu}\right] \backslash$ $D_{\lambda}^{\nu, \rightarrow}:$

$$
\int_{a}^{\widehat{\mu}_{\lambda}(s)} \mu_{\lambda,-}^{*} f d \nu_{\lambda}=\int_{a}^{s} f d \nu_{\lambda}^{\nu}
$$


Proof.

(1) Let $f \in L_{1}\left[\nu_{\lambda},\left[a, b_{\lambda}\right], c\right] \& t \in\left[a, b_{\lambda}\right]$. Then

$$
\begin{aligned}
\int_{a}^{t} f d \nu_{\lambda} & =\int_{a}^{b_{\lambda}} \chi_{[a, t]} \times f d \nu_{\lambda}=\int_{a}^{b_{v}} \widehat{\mu}_{\lambda}^{*}\left(\chi_{[a, t]} \times f\right) d \nu_{\lambda}^{v} \\
& =\int_{a}^{b_{v}}\left(\widehat{\mu}_{\lambda}^{*}\left(\chi_{[a, t]}\right) \times\left(\widehat{\mu}_{\lambda}^{*} f\right) d \nu_{\lambda}^{v},\right.
\end{aligned}
$$

where the first statement in (53) of Theorem 36 was applied and the statement of case (1) in Lemma 30 gave the last equality from which finally we obtain the statement:

$$
\begin{aligned}
\int_{a}^{t} f d \nu_{\lambda} & =\int_{a}^{b_{\nu}}\left(\widehat{\mu}_{\lambda}^{*}\left(\chi_{[a, t]}\right) \times\left(\widehat{\mu}_{\lambda}^{*} f\right) d \nu_{\lambda}^{\nu}\right. \\
& =\int_{a}^{\mu_{\lambda,+}(t)} \widehat{\mu}_{\lambda}^{*} f d \nu_{\lambda}^{\nu} .
\end{aligned}
$$

This proves the first statement.

(2) Let $f \in L_{1}\left[\nu_{\lambda}^{\nu},\left[a, b_{\nu}\right], c\right] \&[a, s] \in \mathscr{B}_{\nu,\left[a, b_{\lambda}\right], c}^{\nu}$ :

$$
\int_{a}^{s} f d \nu_{\lambda}^{v}=\int_{a}^{b_{\nu}} \chi_{[a, s]} \times f d \nu_{\lambda}^{v}=\int_{a}^{b_{\nu}} \mu_{\lambda,-}^{*}\left(\chi_{[a, s]} \times f\right) d \nu_{\lambda}
$$

Now we will apply the statement of case (2) in Lemma 30 to prove the final result:

$$
\begin{aligned}
\int_{a}^{s} f d \nu_{\lambda}^{\nu} & =\int_{a}^{b_{v}} \mu_{\lambda,-}^{*}\left(\chi_{[a, s]} \times f\right) d \nu_{\lambda} \\
& =\int_{a}^{b_{v}}\left(\mu_{\lambda,-}^{*}\left(\chi_{[a, s]}\right) \times\left(\mu_{\lambda,-}^{*} f\right) d \nu_{\lambda}\right. \\
& =\int_{a}^{b_{v}}\left(\chi_{\left[a, \widehat{\mu}_{\lambda}(s)\right]} \times\left(\mu_{\lambda,-}^{*} f\right) d \nu_{\lambda}\right. \\
& =\int_{a}^{\widehat{\mu}_{\lambda}(s)} \mu_{\lambda,-}^{*} f d \nu_{\lambda} .
\end{aligned}
$$

2.2.6. The Main Result. In Theorem 37, we established the relationship between absolute continuous indefinite integrals of members of $L_{1}\left[v_{\lambda},\left[a, b_{\lambda}\right], c\right]$ and the absolute continuous indefinite integrals of members of $L_{1}\left[\nu_{\lambda}^{\nu},\left[a, b_{\nu}\right], c\right]$.

Our next step is to establish the relationship between the indefinite integrals of the members of $L_{1}\left[v,\left[a, b_{\gamma}\right]\right]$ and the indefinite integrals of the members of $\left.L_{1}\left[\nu_{\lambda}^{\nu},\left[a, b_{\nu}\right]\right], c\right]$.

We prove the following statements.

(1) Let $f \in L_{1}\left[\nu,\left[a, b_{\nu}\right]\right]$ and let $\nu_{f, \lambda}(A):=\int_{A} f d \nu, \forall A \in$ $\mathscr{B}_{\lambda,\left[a, b_{y}\right], c^{*}}^{v}$

Let $\nu_{f, \tau}(A):=\int_{A} f d \nu, \forall A \in \mathscr{B}_{\tau,\left[a, b_{\nu}\right], c}^{\nu}$.

(2) From $\mathscr{P}_{\lambda,\left[a, b_{\nu}\right], c}^{\nu} \subset \mathscr{P}\left(\left[a, b_{\nu}\right], \nu\right)$ it follows that $\mathscr{B}_{\lambda,\left[a, b_{\nu}\right], c}^{\nu} \subset \mathscr{B}\left(\left[a, b_{\nu}\right], \nu\right)$.

From $\mathscr{P}_{\tau,\left[a, b_{\nu}\right], c}^{v} \subset \mathscr{P}\left(\left[a, b_{\nu}\right], \nu\right)$ it follows that $\mathscr{B}_{\tau,\left[a, b_{v}\right], c}^{\nu} \subset \mathscr{B}\left(\left[a, b_{\nu}\right], \nu\right)$.
(3) The signed measure $v_{f, \lambda}: \mathscr{B}_{\lambda,\left[a, b_{\nu}\right], c}^{\nu} \longrightarrow R$ is absolute continuous with respect to $v: \mathscr{B}_{\lambda,\left[a, b_{\nu}\right], c}^{\nu} \longrightarrow R^{+}$by $A \in \mathscr{B}_{\lambda,\left[a, b_{\nu}\right], c}^{\nu} \& \nu(A)=0 \Longrightarrow v_{f}(A)=\int_{A} f d \nu=0$.

The signed measure $v_{f, \tau}: \mathscr{B}_{\tau,\left[a, b_{v}\right], c}^{v} \longrightarrow R$ is absolute continuous with respect to $v: \mathscr{B}_{\tau,\left[a, b_{v}\right], c}^{v} \longrightarrow R^{+}$by $A \in \mathscr{B}_{\tau,\left[a, b_{v}\right], c}^{v} \& \nu(A)=0 \Longrightarrow v_{f}(A)=\int_{A} f d v=0$.

(4) By the absolute continuity stated in point (2) mentioned above, the $\mathscr{B}_{\lambda,\left[a, b_{y}\right], c}^{v}$-measurable Radon Nikodym derivative exists:

$\tilde{f}_{v, \lambda}:=\frac{d \nu_{f}}{d \nu_{\lambda}^{\nu}} \Longleftrightarrow \int_{A} f d v=\int_{A} \widetilde{f}_{\nu, \lambda} d \nu_{\lambda}^{\nu}$

$$
\forall A \in \mathscr{B}_{\lambda,\left[a, b_{v}\right], c}^{v}
$$

By the absolute continuity stated in point (2) mentioned above, the $\mathscr{B}_{\tau,\left[a, b_{v}\right], c}^{v}$-measurable Radon Nikodym derivative exists:

$$
\tilde{f}_{\nu, \tau}:=\frac{d \nu_{f}}{d \nu_{\tau}^{\nu}} \Longleftrightarrow \int_{\mathrm{A}} f d \nu=\int_{A} \tilde{f}_{\nu, \tau} d \nu_{\tau}^{\nu},
$$

$$
\forall A \in \mathscr{B}_{\tau,\left[a, b_{\gamma}\right], c}^{v} .
$$

With the statements (1)-(4) mentioned above we have proved the following result.

Theorem 38. A function $f:\left[a, b_{\lambda}\right] \subset R \longrightarrow R^{n}$ is of bounded variation on $\left[a, b_{\lambda}\right]$ if and only if the following conditions are fulfilled.

(1) There exists a closed interval $\left[a, b_{\nu}\right], a, b_{\nu} \in R$ partitioned as union of two disjoint measurable sets $\left[a, b_{\gamma}\right]=$ $N_{\lambda}^{v} \cup N_{\tau}^{v}$ such that $\widehat{\mu}_{\lambda}(s):=a+\int_{a}^{s} \chi_{N_{\lambda}^{v}} d v \in\left[a, b_{\lambda}\right], s \in$ $\left[a, b_{\gamma}\right]$ is onto $\left[a, b_{\lambda}\right]$ (see the details in Definition 15, equation (17)).

(2) There exists an absolute continuous function $\varphi_{f}(u):=$ $f(a)+\int_{a}^{u} \psi_{f} d v:\left[a, b_{\nu}\right] \longrightarrow R^{n}$ with $\psi_{f} \in$ $L_{1}\left[\nu,\left[a, b_{\nu}\right], R^{n}\right]$ such that the following applies.

(a) On the t-scale with the Radon-Nikodym derivative $\widehat{\psi}_{f_{v, \lambda}}$ defined in (64) is expressed by

$$
\begin{aligned}
f(t) & =\varphi_{f}\left(\mu_{\lambda,+}(t)\right)=f(a)+\int_{a}^{\mu_{\lambda,+}(t)} \psi_{f} d \nu \\
& =f(a)+\int_{a}^{\mu_{\lambda,+}(t)} \widehat{\psi}_{f} d \nu_{\lambda}^{\nu} \\
& =f(a)+\int_{a}^{t} \mu_{\lambda,-}^{*} \widehat{\psi}_{f}
\end{aligned}
$$

(b) The $\tau$ scale representation $f_{\tau}$ of $f$ with the Radon - Nikodym derivative $\widehat{\psi}_{f, \tau}$ defined in (65) is expressed by 


$$
\begin{aligned}
f_{\tau}(\vartheta) & =\varphi_{f}\left(\mu_{\tau,+}(\vartheta)\right)=f(a)+\int_{a}^{\mu_{\tau,+}(9)} \psi_{f} d \nu \\
& =f(a)+\int_{a}^{\mu_{\tau,+}(9)} \widehat{\psi}_{f_{\nu, \tau}} d \nu_{\tau}^{\nu} \\
& =f(a)+\int_{a}^{\vartheta} \mu_{\tau,-}^{*} \widehat{\psi}_{f_{\nu, \tau}} d \nu_{\tau}, \quad \forall \vartheta \in\left[0, b_{\tau}\right]
\end{aligned}
$$

where we used the notations and functions defined in Definition 15, equation (17) and the mappings defined in Definitions 16 and 17.

\section{Data Availability}

The data used to support the findings of this study are included within the article.

\section{Conflicts of Interest}

The authors declare that they have no conflicts of interest.

\section{References}

[1] V. Lakshmikantham, D. D. Bainov, and P. S. Simeonov, Theory of Impulsive Differential Equations, World Scientific, Singapore, 1989.

[2] W. Aziz, J. A. Guerrero, and N. Merentes, "On nonlinear integral equations in the space bv()," Journal of Fixed Point Theory and Applications, vol. 18, no. 2, pp. 351-366, 2014.

[3] W. Aziza, H. Leiva, and N. Merentes, "Solutions of Hammerstein equations in the space BV $\left(I_{a}^{b}\right)$," Quaestiones Mathematicae, vol. 37, no. 3, pp. 359-370, 2014.

[4] L. Azocar, H. Leiva, J. Matute, and N. Merentes, "On the hammerstein equationin the space of functions of boundedvariation in the plane," Archivum Mathematicum, vol. 49, no. 1, pp. 51-64, 2013.

[5] A. C. Ponce and D. Spector, "On formulae decoupling the total variation of BV functions," Nonlinear Analysis, vol. 154, pp. 241257, 2017.

[6] P. Umberto, "Singular continuous functions. Electronic. Real Analysis," 2014.

[7] J. A. Clarkson and C. R. Adams, "On definitions of bounded variation for functions of two variables," Transactions of the American Mathematical Society, vol. 35, no. 4, pp. 824-854, 1933.

[8] P. R. Halmos, Measure Theory, Springer, New York, NY, USA, 1950.

[9] J. Yeh, Real Analysis. Theory of Measure and Integration, World Scientific Publishing Co. Pte. Ltd., Hackensack, NJ, USA, Second edition, 2006.

[10] R. F. Bass, "Measure and integration theory," Article ID 9781466391574, ISBN-13 978-1466391574, ISBN-10 146639157X, 2011.

[11] H. L. Royden and P. M. Fitzpatrick, Real Analysis, Pearson Education Asia Limited and China Machine Press - Prentice Hall, 4th edition, 2010, Number ISBN 978-0-13-143747-0. 


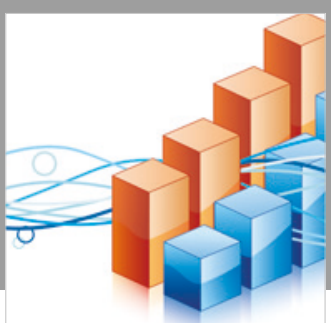

Advances in

Operations Research

\section{-n-m}
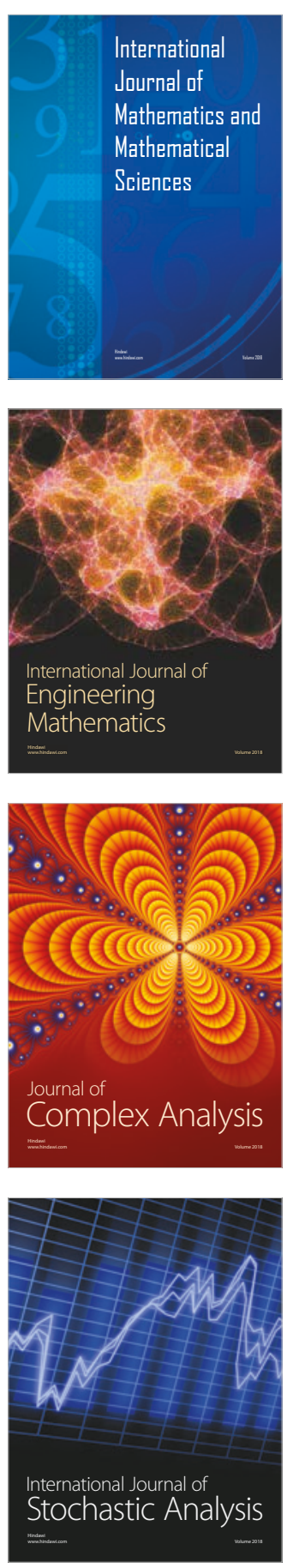
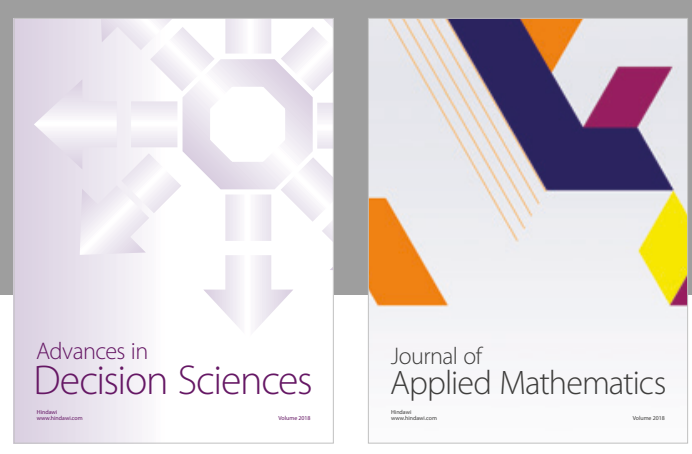

Journal of

Applied Mathematics
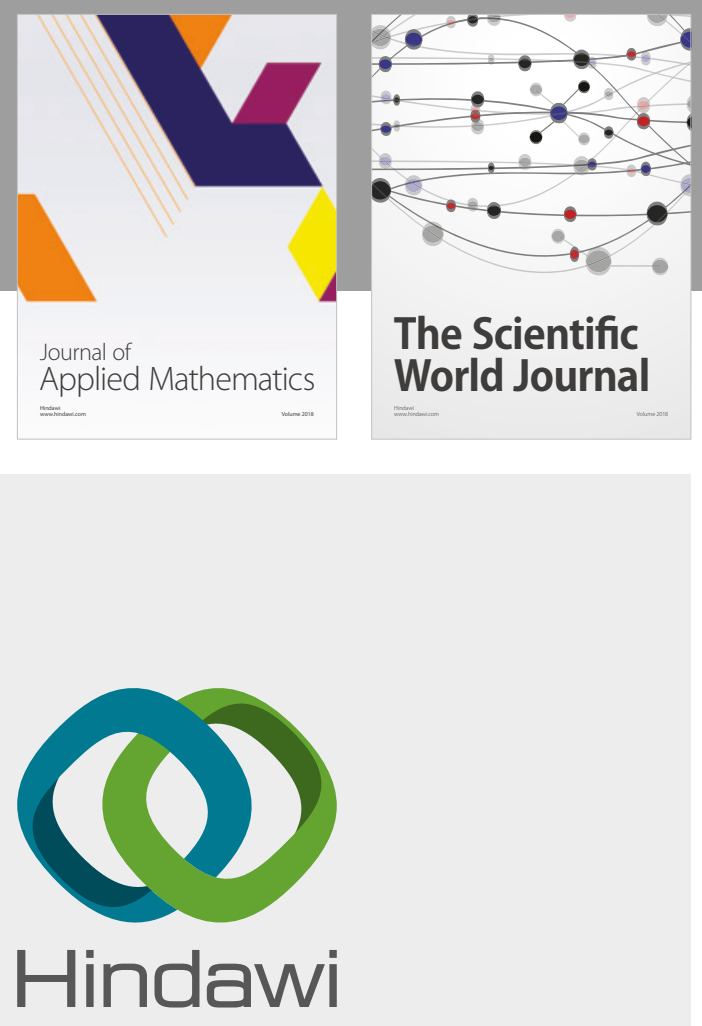

Submit your manuscripts at

www.hindawi.com

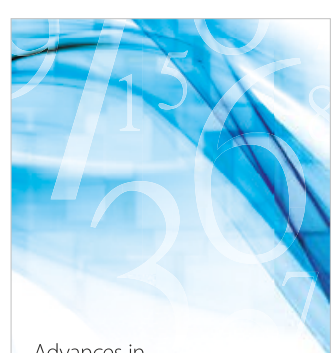

Advances in
Numerical Analysis
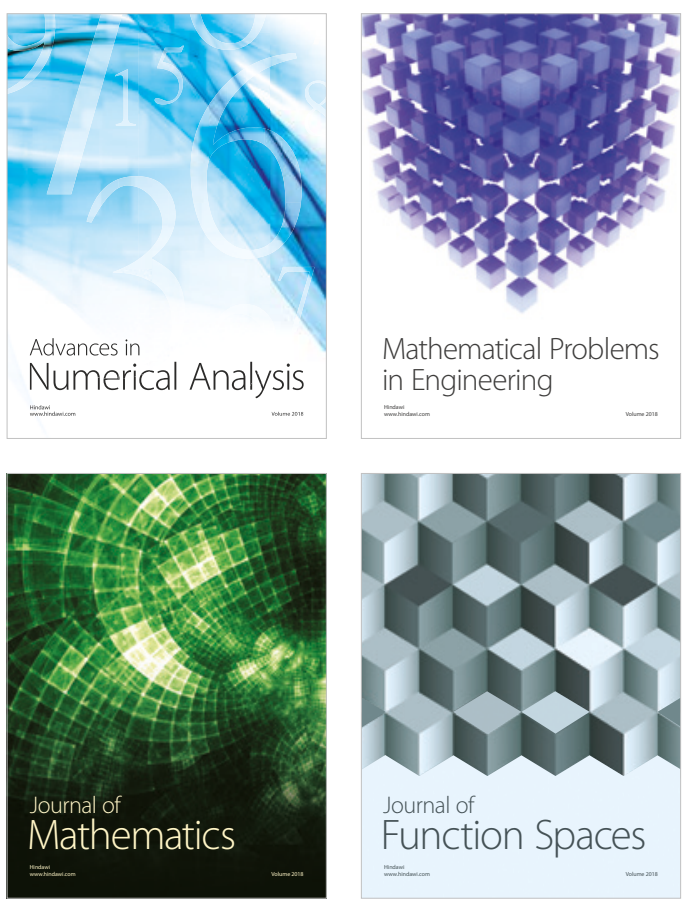

Mathematical Problems in Engineering

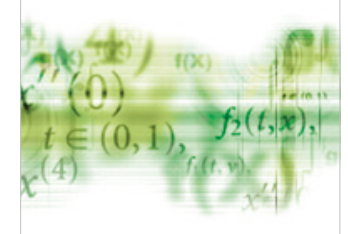

International Journal of

Differential Equations

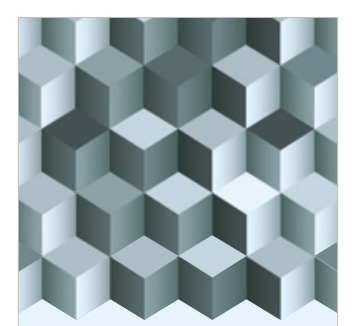

Journal of

Function Spaces
The Scientific

World Journal

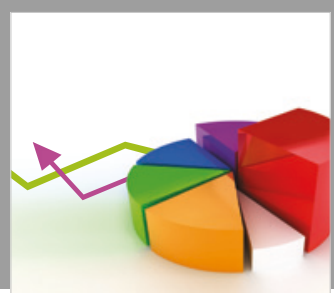

Journal of

Probability and Statistics
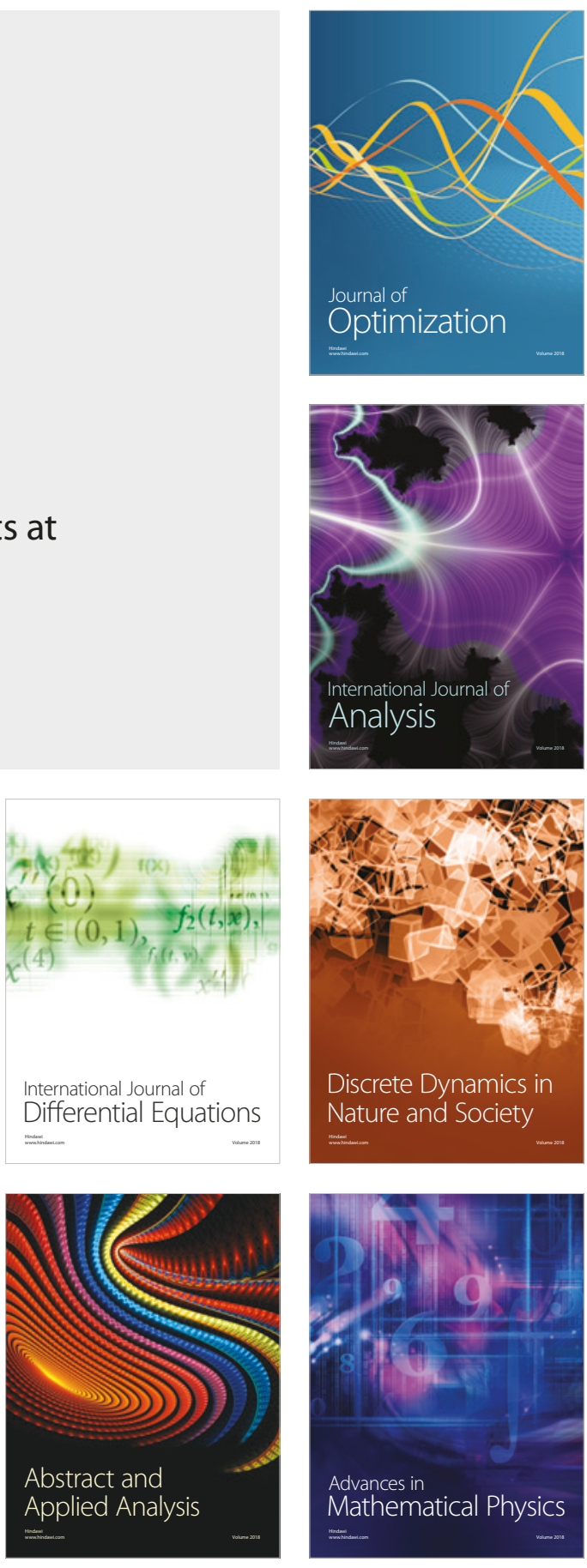